\title{
Deformations of Hypercomplex Structures associated to Heisenberg Groups
}

\author{
Gueo Grantcharov * Henrik Pedersen ${ }^{\dagger}$ Yat Sun Poon ${ }^{\ddagger}$ \\ September 10, 2018
}

\begin{abstract}
Let $X$ be a compact quotient of the product of the real Heisenberg group $H_{4 m+1}$ of dimension $4 m+1$ and the 3 -dimensional real Euclidean space $\mathbf{R}^{3}$. A left invariant hypercomplex structure on $H_{4 m+1} \times \mathbf{R}^{3}$ descends onto the compact quotient $X$. The space $X$ is a hyperholomorphic fibration of 4 -tori over a $4 m$-torus. We calculate the parameter space and obstructions to deformations of this hypercomplex structure on $X$. Using our calculations we show that all small deformations generate invariant hypercomplex structures on $X$ but not all of them arise from deformations of the lattice. This is in contrast to the deformations on the $4 m$-torus.
\end{abstract}

Keywords: Heisenberg group, nilpotent groups, Kodaira surfaces, Kodaira manifolds, hypercomplex, deformation.

AMS Subject Classification: Primary 32G07. Secondary 53C15, 53C56, 32L25, 57S25.

\section{Introduction}

Heisenberg groups play a fundamental role in many branches of mathematics. One of its appearances is in the construction of Kodaira surfaces. Among other features, these surfaces can be realized as an elliptic fibration over elliptic curves. This is a realization of the quotient map from the Heisenberg group with respect to its center.

*Permanent Address: Department of Mathematics, Florida International University, Miami, FL 33199, Partially supported by NSF DMS-0333172

${ }^{\dagger}$ Address: Department of Mathematics and Computer Science, University of Southern Denmark, Campusvej 55, Odense M, DK-5230, Denmark. E-mail: henrik@adm.sdu.dk. Partially supported by the European contract HPRN-CT-2000-00101.

$\ddagger$ Address: Department of Mathematics, University of California at Riverside, Riverside, CA 92521, U.S.A.. E-mail: ypoon@math.ucr.edu. Partially supported by NSF DMS-0204002. 
This construction is extended to higher dimensions in [6] [12]. On the other hand, Heisenberg groups are used by several authors to construct hypercomplex structures [9] [3] [1. This direction greatly enriches the source of hypercomplex manifolds as the past constructions of compact examples are often limited to homogeneous spaces with semi-simple Lie groups [8] [16]. The construction and the deformations of these hypercomplex structures are the topics of this article.

Let $H_{2 n+1}$ be the $2 n+1$-dimensional real Heisenberg group. It is the extension of the Abelian additive group $\mathbf{R}^{2 n}$ by a one-dimensional center. The space $\mathbf{R}^{2 n}$ is a complex space as it can be real linearly identified to the complex vector space $\mathbf{C}^{n}$. The product $H_{2 n+1} \times \mathbf{R}^{1}$ admits a left-invariant complex structures such that the natural projection $\phi$ onto $\mathbf{R}^{2 n}$ is holomorphic. Taking compact quotients, we obtain a complex structure on a generalization of Kodaira manifolds $X$ [6]. This construction can be extended further to a construction of hypercomplex structure.

The quotient of the $4 m+1$-dimensional real Heisenberg group $H_{4 m+1}$ with its one-dimensional center is the Abelian additive group $\mathbf{R}^{4 m}$. The space $\mathbf{R}^{4 m}$ is a hypercomplex space when it is identified to the module of quaternions $\mathbf{H}^{m}$. The product $H_{4 m+1} \times \mathbf{R}^{3}$ admits a left-invariant hypercomplex structures such that the natural projection $\phi$ onto $\mathbf{R}^{4 m}$ is hyper-holomorphic. Taking compact quotients $X$, we obtain a hypercomplex manifold fibered over the torus $T^{4 m}$ with its standard hypercomplex structures. The fiber is a four-dimensional torus $T^{4}$ obtained as a compact quotient of the product of the group $H_{4 m+1} \times \mathbf{R}^{3}$. In this paper, we first calculate the parameter spaces and obstructions to deformation of the hypercomplex structure on the compact quotient $X$ of $H_{4 m+1} \times \mathbf{R}^{3}$. Our computation is based on twistor theory, deformation theory of maps and an understanding of the deformation of hypercomplex structures on the torus. Then we compare this parameter space with the space of the invariant hypercomplex structures and the deformation space arising from the deformations of the lattice in $H_{4 m+1} \times \mathbf{R}^{3}$.

In Section 1, we explain the relations among twistor theory, deformation theory of holomorphic maps and deformation theory of hypercomplex manifolds. Through these relations, we compute the parameter space for hypercomplex deformations in Section 2. The next theorem is the result of enumerating the dimension of the parameter space in a long exact sequence of cohomology.

Theorem 1 The real dimension of the virtual parameter space of deformations of hypercomplex structures on the $(4 m+4)$-dimensional manifold $X$ is equal to $6 m^{2}+$ $11 m+12$.

In the parameter space, there is a twelve-dimensional subspace contributed by the deformation of the 4-torus in the fiber of the projection $\phi$. Hypercomplex deformations of the base of this projection contribute to a subspace of dimension $3\left(2 m^{2}+m\right)$. In Lemma 3, we establish that the dimension of the parameter space of hypercomplex 
structures on a torus of dimension $4 m$ is naturally identified to $12 m^{2}$ and that only some of the hypercomplex deformation on the base torus comes from a hypercomplex deformation on the Kodaira manifold. Since the space of obstructions to deformation does not vanish, we study the integrability of deformation parameters by constructing convergent power series in Section 3.

Theorem 2 Every point in the virtual parameter space is an infinitesimal deformation of an integrable deformation.

In this construction, we do not control the power series enough to claim a priori that the deformation must be hypercomplex. At this point we produce a deformation of quaternionic structures only. We conclude our work showing that the deformed twistor spaces have a holomorphic projection onto $\mathbf{C P}^{1}$. Through the twistor correspondence, we complete a proof of the following theorem.

Theorem 3 Every quaternionic deformation of the hypercomplex structure on $X$ is a hypercomplex deformation.

This observation raises the issue of enumerating the number of parameters for quaternionic deformations of the hypercomplex manifold $X$. This is achieved through a coboundary map computation.

Theorem 4 The real dimension of the parameter space of deformations of quaternionic structures on the $(4 m+4)$-dimensional manifold $X$ is equal to $6 m^{2}+11 m+9$.

During the course of our computation, we reveal a considerable amount of information about the deformation of hypercomplex structures on the torus $T^{4 m}$, because its twistor space $Z$ is the base space of an elliptic fibration from the twistor space $W$ of the hypercomplex structure $X$. In section 4.5 we reveal how our computation may be used to identify the moduli space of hypercomplex structures on a torus.

Finally in Section 5, we calculate the parameter space of the invariant hypercomplex structures on $X$ and space of deformations arising from the deformations of the lattice. Although in Section 3 we do not control the power series completely, the information we obtain there is enough to prove the first part of the following:

Theorem 5 Any small deformation of the hypercomplex structure on $X$ consists of invariant structures. However there are deformations which do not arise from a deformation of the lattice $\Gamma$.

The second part of Theorem 5 follows by direct dimension count. The proof is at the end of Section 5 . 


\section{Basic Constructions}

\subsection{The Heisenberg group and the Heisenberg algebra}

The real Heisenberg group of dimensional $4 \mathrm{~m}+1$ is the Lie group $H_{4 m+1}$ whose underlying manifold is $\mathbf{R}^{4 m} \times \mathbf{R}$ with coordinates $(x, y, z):=\left(x_{1}, \ldots, x_{2 m}, y_{1}, \ldots, y_{2 m}, z\right)$ and whose group law is given by

$$
(x, y, z) *\left(x^{\prime}, y^{\prime}, z^{\prime}\right)=\left(x+x^{\prime}, y+y^{\prime}, z+z^{\prime}-2 \sum_{j=1}^{2 m}\left(x_{j} y_{j}^{\prime}-y_{j} x_{j}^{\prime}\right)\right) .
$$

The left translations of $\left\{\frac{\partial}{\partial x_{j} \mid \mathbf{0}}, \frac{\partial}{\partial y_{j}},\left.\frac{\partial}{\partial z}\right|_{0}\right\}$ are the following vector fields.

$$
X_{j}=\frac{\partial}{\partial x_{j}}+2 y_{j} \frac{\partial}{\partial z}, \quad Y_{j}=\frac{\partial}{\partial y_{j}}-2 x_{j} \frac{\partial}{\partial z}, \quad Z=\frac{\partial}{\partial z} .
$$

These vectors form a basis for the Heisenberg algebra $\mathfrak{h}_{4 m+1}$ of the Heisenberg group $H_{4 m+1}$. The commutation relations are as follows:

$$
\left[Y_{j}, X_{k}\right]=4 \delta_{j k} Z, \quad\left[X_{j}, X_{k}\right]=\left[Y_{j}, Y_{k}\right]=\left[X_{j}, Z\right]=\left[Y_{j}, Z\right]=0 .
$$

The subspace $\mathfrak{c}$ spanned by $Z$ is the center of the Heisenberg algebra. The quotient space of the Heisenberg algebra with respect to the center is the $4 m$-dimensional Abelian algebra $\mathfrak{t}_{4 m}$. Therefore, we have the exact sequence

$$
0 \rightarrow \mathfrak{c} \stackrel{\iota}{\rightarrow} \mathfrak{h}_{4 m+1} \stackrel{\phi}{\rightarrow} \mathfrak{t}_{4 m} \rightarrow 0 .
$$

On the level of Lie groups, we have a group homomorphism

$$
\phi: H_{4 m+1}=\left(\mathbf{R}^{4 m+1}, *\right) \rightarrow\left(\mathbf{R}^{4 m},+\right)
$$

obtained as the quotient of the central subgroup $C=\left(\mathbf{R}^{1},+\right)$. Although it is obvious that

$$
\phi\left(X_{j}\right)=\frac{\partial}{\partial x_{j}}, \quad \phi\left(Y_{j}\right)=\frac{\partial}{\partial y_{j}}, \quad \phi(Z)=\frac{\partial}{\partial z},
$$

it will be important for our future computation that these identities give a way to lift vector fields from the Abelian group to the Heisenberg group.

Let $\Gamma$ be the subgroup $\left(\mathbf{Z}^{4 m+1}, *\right)$ of the Heisenberg group. The intersection $\Gamma_{0}$ of $\Gamma$ with the central subgroup is isomorphic to the integer group $\mathbf{Z}$. The quotient of $C$ by $\Gamma_{0}$ is the one-dimensional torus group $T^{1}$. The quotient of the additive Abelian group $\mathbf{R}^{4 m}$ by $\phi(\Gamma)$ is the $4 \mathrm{~m}$-dimensional torus group $T^{4 m}$. It is obvious 
that the homomorphism $\iota$ from the central subgroup $C$ into the Heisenberg group $H_{4 m+1}$ intertwines $\Gamma_{0}$ and $\Gamma$, and the homomorphism $\phi$ from the Heisenberg group $H_{4 m+1}$ to the Abelian group $\mathbf{R}^{4 m}$ intertwines the groups $\Gamma$ and $\phi(\Gamma)$. Therefore, the homomorphisms $\iota$ and $\phi$ descend to maps between compact quotients. Since $C$ is the central subgroup, its action commutes with the action of the lattice $\Gamma$. Therefore, the quotient group $T^{1}$ acts on the quotient space of left-cosets $\Gamma \backslash H_{4 m+1}$. The orbits of this group action are precisely the fibers of the projection

$$
\phi: \Gamma \backslash H_{4 m+1} \rightarrow T^{4 m}
$$

From now on, we denote the quotient space $\Gamma \backslash H_{4 m+1}$ by $\check{H}_{4 m+1}$ or $\check{H}$ if the dimension of the group is clear in a given context.

\subsection{A Construction of Hypercomplex Structures}

Three complex structures $I_{1}, I_{2}$ and $I_{3}$ on a smooth manifold form a hypercomplex structure if

$$
I_{1}^{2}=I_{2}^{2}=I_{3}^{2}=-I_{0}, \quad \text { and } \quad I_{1} I_{2}=I_{3}=-I_{2} I_{1},
$$

where $I_{0}$ is the identity map.

Let $\mathfrak{t}_{3}$ be the 3-dimensional Abelian algebra. The direct sum $\mathfrak{h}_{4 m+1} \oplus \mathfrak{t}_{3}$ is a 2-step nilpotent algebra whose center is four-dimensional. Fix a basis $\left\{E_{1}, E_{2}, E_{3}\right\}$ for $\mathfrak{t}_{3}$. Consider the endomorphisms $I_{1}, I_{2}$ and $I_{3}$ of $\mathfrak{h}_{4 m+1} \oplus \mathfrak{t}_{3}$ defined by left multiplications

of the quaternions $i, j$ and $k$ on the module of quaternions $\mathbf{H}$, and the identifications

$$
\begin{aligned}
x_{2 a-1} X_{2 a-1}+x_{2 a} X_{2 a}+y_{2 a-1} Y_{2 a-1}+y_{2 a} Y_{2 a} & \rightarrow x_{2 a-1}+x_{2 a} i+y_{2 a-1} j+y_{2 a} k \\
z Z+t_{1} E_{1}+t_{2} E_{2}+t_{3} E_{3} & \rightarrow z+t_{1} i+t_{2} j+t_{3} k .
\end{aligned}
$$

In other words, for $1 \leq a \leq m$,

$$
\begin{array}{rlll}
I_{1} X_{2 a-1}=X_{2 a}, & I_{1} Y_{2 a-1}=Y_{2 a}, & I_{1} Z=E_{1}, & I_{1} E_{2}=E_{3} ; \\
I_{2} X_{2 a-1}=Y_{2 a-1}, & I_{2} X_{2 a}=-Y_{2 a}, & I_{2} Z=E_{2}, & I_{2} E_{1}=-E_{3} \\
I_{3} X_{2 a-1}=Y_{2 a}, & I_{3} X_{2 a}=Y_{2 a-1}, & I_{3} Z=E_{3}, & I_{3} E_{1}=E_{2} .
\end{array}
$$

Through left translations, these endomorphisms define almost complex structures on the product of the Heisenberg group and the three-dimensional additive group $H_{4 m+1} \times \mathbf{R}^{3}$. By construction, these almost complex structures satisfy the algebra (8). As $\left[I_{a} X, I_{a} Y\right]=[X, Y]$ for any left-invariant vector fields $X$ and $Y$ and $1 \leq a \leq 3$, these complex structures are integrable. It implies that $\left\{I_{a}: a=1,2,3\right\}$ is a leftinvariant hypercomplex structure on the Lie group $H_{4 m+1} \times \mathbf{R}^{3}$. Let $\mathbf{Z}^{3}$ be the integer subgroup of $\mathbf{R}^{3}$. Then the quotient space $\check{H} \times \mathbf{R}^{3} / \mathbf{Z}^{3}$ is the compact manifold $\check{H} \times T^{3}$. 
We denote this compact hypercomplex manifold by $X$. The natural projection $\phi$ from $X$ onto $T^{4 m}$ and the inclusion $\iota$ from $T^{1} \times T^{3} \cong T^{4}$ into $X$ are both hyper-holomorphic maps. The fibers of the projection $\phi$ are the orbits of the left-action of $T^{1} \times T^{3}$.

For the hypercomplex structure we show:

Theorem 6 The hypercomplex structure on $H_{4 m+1} \times \mathbf{R}^{3}$ constructed above is equivalent to the standard one on $\mathbf{H}^{m+1}$.

Proof: From the definition of the vector fields $X_{i}, Y_{i}, Z, E_{i}$ we see that the dual 1 -forms are $d x_{i}, d y_{i}, \theta=d z-2 \Sigma\left(y_{i} d x_{i}-x_{i} d y_{i}\right), d e_{i}$, where $e_{1}, e_{2}, e_{3}$ are coordinates of $\mathbf{R}^{3}$. Then we have the same identities for the action of the hypercomplex structure on the 1 -forms as we had for the vector fields, e.g. $I_{1} d x_{1}=d x_{2} \ldots$ etc. With this in mind we calculate:

$$
\begin{aligned}
I_{1} d z & =I_{1} \theta+I_{1} 2 \sum_{i}\left(y_{i} d x_{i}-x_{i} d y\right)= \\
& =d e_{1}+2 \sum_{a}\left(y_{2 a-1} d x_{2 a}-x_{2 a-1} d y_{2 a}-y_{2 a} d x_{2 a-1}+x_{2 a} d y_{2 a-1}\right) \\
& =d\left(e_{1}+2 \sum_{a}\left(y_{2 a-1} x_{2 a}-x_{2 a-1} y_{2 a}\right)\right)
\end{aligned}
$$

so by defining

$$
f_{1}:=e_{1}+2 \sum_{a}\left(y_{2 a-1} x_{2 a}-x_{2 a-1} y_{2 a}\right)
$$

we have $I_{1} d z=d f_{1}$. Similarly,

$$
\begin{aligned}
I_{2} d z & =I_{2} \theta+I_{2} 2 \sum_{i}\left(y_{i} d x_{i}-x_{i} d y\right)= \\
& \left.=d e_{2}+2 \sum_{a}\left(y_{2 a-1} d y_{2 a-1}+x_{2 a-1} d x_{2 a-1}-y_{2 a} d y_{2 a}-x_{2 a} d x_{2 a}\right)\right) \\
& =d\left(e_{2}+\sum_{a}\left(y_{2 a-1}^{2}+x_{2 a-1}^{2}-y_{2 a}^{2}-x_{2 a}^{2}\right)\right)
\end{aligned}
$$

so $I_{2} d z=d f_{2}$, for

$$
f_{2}:=e_{2}+\sum_{a}\left(y_{2 a-1}^{2}+x_{2 a-1}^{2}-y_{2 a}^{2}-x_{2 a}^{2}\right)
$$

Finally, by similar calculation we have $I_{3} d z=d f_{3}$, where

$$
f_{3}=e_{3}+2 \sum_{a}\left(y_{2 a-1} y_{2 a}+x_{2 a-1} x_{2 a}\right)
$$

Now from here we have that $x_{i}, y_{i}, z, f_{i}$ are quaternionic coordinates, which are global on $\mathbf{R}^{4 m+4}$, so identify our hypercomplex structure with the standard one as claimed. q.e.d.

The above calculation could be done using the Obata connection and then one can show that $d f_{i}$ are parallel 1-forms. Here we outline the argument. The Obata connection is given by

$$
\nabla_{X} Y=1 / 2[X, Y]+1 / 12 \sum_{i, j, k} I_{i}\left(\left[I_{j} X, I_{k} Y\right]+\left[I_{j} Y, I_{k} X\right]\right)+1 / 6 \sum_{i} I_{i}\left(\left[I_{i} X, Y\right]+\left[I_{i} Y, X\right]\right)
$$


for hypercomplex manifold, where $(i, j, k)$ is cyclic permutation of $(1,2,3)$. Then for abelian hypercomplex structure this reduces to

$$
\nabla_{X} Y=1 / 2[X, Y]+1 / 2 \sum_{i} I_{i}\left[I_{i} X, Y\right]
$$

From here one has that $d x_{i}, d y_{i}$ are parallel and $\nabla \theta=1 / 2 d \theta$. Now from $\nabla\left(x_{i} d y_{j}\right)=$ $d x_{i} \otimes d y_{j}$ one easily checks that $d z$ and $d f_{i}$ are parallel.

\subsection{Twistor Theory}

We identify points $\vec{a}=\left(a_{1}, a_{2}, a_{3}\right)$ in the unit 2-sphere $S^{2}$ to the complex structure $I_{\vec{a}}=a_{1} I_{1}+a_{2} I_{2}+a_{3} I_{3}$. Let $J_{\vec{a}}$ be the complex structure on $S^{2}$ defined by the stereographic projection

$$
\mu \in \mathbf{C} \mapsto \vec{a}=\frac{1}{1+|\mu|^{2}}\left(|\mu|^{2}-1,-i(\mu-\bar{\mu}), \mu+\bar{\mu}\right) .
$$

This map takes 0 to $-I_{1}, i$ to $I_{2}$ and 1 to $I_{3}$. It sends the complex orientation of the complex plane to the outward normal orientation of the sphere. Therefore, the complex structure on the sphere at the unit vector $\vec{a}$ is defined by the cross product with $\vec{a}$.

The smooth manifold $W=X \times S^{2}$ is endowed with an almost complex structure $\mathcal{I}$ defined by $\mathcal{I}_{(x, \vec{a})}=I_{\vec{a}} \oplus J_{\vec{a}}$. By twistor theory, this is an integrable complex structure [15]. Moreover, the projection $p$ from $W$ onto $S^{2}$ is a holomorphic projection such that the fiber $p^{-1}(\vec{a})$ is the complex manifold $\left(X, I_{\vec{a}}\right)$. The holomorphic projection from $Z$ onto $\mathbf{C P}^{1}$ is also denoted by $p$. The map is also real in the sense that there is an anti-holomorphic involution $\tau$ on the twistor space $W$ such that $p \circ \tau=\rho \circ p$ where $\rho$ is the anti-podal map on the 2 -sphere.

As explained in [16], deformations of hypercomplex structures are identified to deformations of the real map $p$. Deformations of this map $p$ are described by the

cohomology spaces $H^{k}\left(W, \mathcal{D}_{W}\right)$ where $\mathcal{D}_{W}$ is the kernel of the differential $d p$ [7]. The real part of these spaces contain the deformation of the hypercomplex structures. Since there is also a correspondence between quaternionic structure and the complex structures on the twistor space [18], the real part of the cohomology spaces $H^{k}\left(W, \Theta_{W}\right)$ contains the deformation theory of the quaternionic structures.

\section{Deformation Parameters of $X=\check{H} \times T^{3}$}

The aim of this section is to compute the cohomology spaces $H^{k}\left(W, \mathcal{D}_{W}\right)$ of the twistor space $W$ for the hypercomplex manifold $X$. We begin with some standard computation on the twistor space $Z$ over the torus. 


\subsection{Twistor Space of the Torus}

The twistor space $Z$ is the quotient of the bundle $\mathfrak{t}^{1,0} \otimes \mathcal{O}(1)$ on $\mathbf{C P}^{1}$ where $\mathfrak{t}^{1,0}$ is the (1,0)-part of the complexification of the $4 m$-dimensional Abelian algebra $\mathfrak{t}[2$, Example 13.64 and Example 13.66].

Choose linear coordinates $\left(z_{1}^{a}, z_{2}^{a}, z_{1}, z_{2}\right), a=1, \ldots, m$, for $\mathbf{C}^{2 m+2}$. They are related to real coordinates of $H_{4 m+1} \times \mathbf{R}^{3}$ by

$$
z_{1}^{a}=x_{2 a-1}+i x_{2 a}, \quad z_{2}^{a}=y_{2 a-1}+i y_{2 a}, \quad z_{1}=z+i t_{1}, \quad z_{2}=t_{2}+i t_{3} .
$$

Let $\left[\lambda_{1}, \lambda_{2}\right]$ be the homogeneous coordinates on $\mathbf{C P}^{1}$. On $U_{1}=\left\{\lambda \in \mathbf{C P}^{1}: \lambda_{1} \neq\right.$ $0\}$, define $\nu=\frac{\lambda_{2}}{\lambda_{1}}$. On $U_{2}=\left\{\lambda \in \mathbf{C P}^{1}: \lambda_{2} \neq 0\right\}$, define $\mu=\frac{\lambda_{1}}{\lambda_{2}}$. We use the same notation to denote $p^{-1}\left(U_{1}\right)$ and $p^{-1}\left(U_{2}\right)$ on both $Z$ and $W$.

Lemma 1 Let $\mathcal{O}$ be the structure sheaf of the twistor space $Z$. Let $R^{q} p_{*} \mathcal{O}$ be the $q$-th direct image sheaf with respect to the projection $p$ from $Z$ onto $\mathbf{C P}^{1}$. Then

$$
R^{q} p_{*} \mathcal{O}=\wedge^{q}\left(\mathfrak{t}^{*(0,1)} \otimes \mathcal{O}(1)\right)
$$

Proof: Since the dimension of $H^{q}\left(p^{-1}(\lambda), \mathcal{O}\right)$ is constant with respect to $\lambda$, the direct image sheaves $R^{q} p_{*} \mathcal{O}$ are locally free. As each fiber $p^{-1}(\lambda)$ is an Abelian variety, $H^{q}\left(p^{-1}(\lambda), \mathcal{O}\right)=\wedge^{q} H^{1}\left(p^{-1}(\lambda), \mathcal{O}\right)$, and there is a vector bundle isomorphism $R^{q} p_{*} \mathcal{O}=\wedge^{q} R^{1} p_{*} \mathcal{O}$.

On $U_{2}$, for $1 \leq a \leq m$, the (0,1)-forms

$$
\bar{\sigma}_{1}^{a}=\frac{\bar{\mu} d \bar{z}_{1}^{a}-d z_{2}^{a}}{1+|\mu|^{2}}, \quad \bar{\sigma}_{2}^{a}=\frac{\bar{\mu} d \bar{z}_{2}^{a}+d z_{1}^{a}}{1+|\mu|^{2}}
$$

are holomorphic because

$$
d \bar{\sigma}_{1}^{a}=\frac{1}{1+|\mu|^{2}}\left(d \bar{\mu} \wedge \sigma_{2}^{a}-\bar{\mu} d \mu \wedge \bar{\sigma}_{1}^{a}\right), \quad d \bar{\sigma}_{2}^{a}=\frac{1}{1+|\mu|^{2}}\left(-\bar{\mu} d \mu \wedge \bar{\sigma}_{2}^{a}-d \bar{\mu} \wedge \sigma_{1}^{a}\right)
$$

are type $(1,1)$-forms. Since for every $\lambda, h^{1}\left(p^{-1}(\lambda), \mathcal{O}\right)=2 m$, these holomorphic forms determine a trivialization of the bundle $R^{1} p_{*} \mathcal{O}\left(U_{2}\right)$. Similarly, on $U_{1}$ the $(0,1)$-forms

$$
\bar{\rho}_{1}^{a}=\frac{d \bar{z}_{1}^{a}-\bar{\nu} d z_{2}^{a}}{1+|\nu|^{2}}, \quad \bar{\rho}_{2}^{a}=\frac{d \bar{z}_{2}^{a}+\bar{\nu} d z_{1}^{a}}{1+|\nu|^{2}}
$$

determine a holomorphic trivialization of the bundle $R^{1} p_{*} \mathcal{O}\left(U_{1}\right)$. As $\bar{\rho}_{i}^{a}=\mu \bar{\sigma}_{i}^{a}$, the bundle $R^{1} p_{*} \mathcal{O}$ is isomorphic to $\mathfrak{t}^{*(0,1)} \otimes \mathcal{O}(1)$ as claimed. q. e. d. 
Lemma 2 For $\ell \geq-1, H^{k}\left(Z, p^{*} \mathcal{O}(\ell)\right)=\mathfrak{t}^{*(0, k)} \otimes S^{\ell+k} \mathbf{C}^{2}$ where $S^{j} \mathbf{C}^{2}$ is the $j$-th symmetric tensor product of $\mathbf{C}^{2}$.

Proof: The projection formula and the last lemma determine the isomorphism

$$
R^{q} p_{*} p^{*} \mathcal{O}(\ell)=R^{q} p_{*} \mathcal{O} \otimes \mathcal{O}(\ell)=\mathcal{O}(\ell) \otimes \wedge^{q}\left(\mathfrak{t}^{*(0,1)} \otimes \mathcal{O}(1)\right)=\mathcal{O}(\ell+q) \otimes \mathfrak{t}^{*(0, q)} .
$$

Consider the Leray spectral sequence with $E_{2}^{p, q}=H^{p}\left(\mathbf{C P}^{1}, R^{q} p_{*} p^{*} \mathcal{O}(\ell)\right)$, and $E_{\infty}^{p, q} \Rightarrow$ $H^{p+q}\left(Z, p^{*} \mathcal{O}(\ell)\right)$. When $\ell \geq-1, E_{2}^{p, q}=0$ for all $p \geq 1$ and $q \geq 0$. Therefore, the spectral sequence degenerates at $E_{2}$, and

$$
\begin{aligned}
H^{k}\left(Z, p^{*} \mathcal{O}(\ell)\right) & =\oplus_{p+q=k} E_{2}^{p, q}=E_{2}^{0, k}=H^{0}\left(\mathbf{C P}^{1}, R^{k} p_{*} p^{*} \mathcal{O}(\ell)\right) \\
& =H^{0}\left(\mathbf{C P}^{1}, \mathcal{O}(\ell+k) \otimes \mathfrak{t}^{*(0, k)}\right)=\mathfrak{t}^{*(0, k)} \otimes S^{\ell+k} \mathbf{C}^{2} .
\end{aligned}
$$

q. e. d.

The cohomology spaces of the last lemma can be described explicitly. Define

$$
\bar{\Omega}_{1}^{a}=\frac{\bar{\lambda}_{1} d \bar{z}_{1}^{a}-\bar{\lambda}_{2} d z_{2}^{a}}{\left|\lambda_{1}\right|^{2}+\left|\lambda_{2}\right|^{2}}, \quad \bar{\Omega}_{2}^{a}=\frac{\bar{\lambda}_{1} d \bar{z}_{2}^{a}+\bar{\lambda}_{2} d z_{1}^{a}}{\left|\lambda_{1}\right|^{2}+\left|\lambda_{2}\right|^{2}}
$$

Then $\left\{\lambda_{1} \bar{\Omega}_{1}^{a}, \lambda_{2} \bar{\Omega}_{1}^{a}, \lambda_{1} \bar{\Omega}_{2}^{a}, \lambda_{2} \bar{\Omega}_{2}^{a}\right\}$ forms a basis for the space $H^{1}(Z, \mathcal{O})$. More generally, the space $H^{k}\left(Z, p^{*} \mathcal{O}(\ell)\right)$, for $\ell \geq 0$ is spanned by the twisted $\mathrm{k}$-forms

$$
\left(\lambda_{1}^{\ell+k-l} \lambda_{2}^{l}\right) \bar{\Omega}_{1}^{a_{1}} \wedge \cdots \wedge \bar{\Omega}_{1}^{a_{i}} \wedge \bar{\Omega}_{2}^{b_{1}} \wedge \cdots \wedge \bar{\Omega}_{2}^{b_{j}}
$$

where $0 \leq l \leq \ell+k$. The space $H^{1}(Z, \mathcal{O})$ has an alternative description. For $k=0,1,2,3$ and over $p^{-1}(\vec{a})$, define 1 -forms

$$
\bar{\omega}_{k}^{a}=I_{k} d x_{2 a-1}-i I_{\vec{a}} I_{k} d x_{2 a-1} .
$$

These $(0,1)$-forms on the twistor space span the space $H^{1}(Z, \mathcal{O})$ because

$$
\begin{array}{ll}
\bar{\omega}_{0}^{a}=\mu \bar{\sigma}_{1}^{a}+\bar{\sigma}_{2}^{a}=\lambda_{1} \bar{\Omega}_{1}^{a}+\lambda_{2} \bar{\Omega}_{2}^{a}, & \bar{\omega}_{1}^{a}=i\left(\mu \bar{\sigma}_{1}^{a}-\bar{\sigma}_{2}^{a}\right)=i\left(\lambda_{1} \bar{\Omega}_{1}^{a}-\lambda_{2} \bar{\Omega}_{2}^{a}\right), \\
\bar{\omega}_{2}^{a}=\mu \bar{\sigma}_{2}^{a}-\bar{\sigma}_{1}^{a}=\lambda_{1} \bar{\Omega}_{2}^{a}-\lambda_{2} \bar{\Omega}_{1}^{a}, & \bar{\omega}_{3}^{a}=i\left(\bar{\sigma}_{1}^{a}+\mu \bar{\sigma}_{2}^{a}\right)=i\left(\lambda_{1} \bar{\Omega}_{2}^{a}+\lambda_{2} \bar{\Omega}_{1}^{a}\right) .
\end{array}
$$

The differential $d p$ from the tangent sheaf to the pull-back of the tangent sheaf $p^{*} \mathcal{O}(2)$ on the projective line $\mathbf{C P}^{1}$ is the twisted 1-form $d p=\lambda_{2} d \lambda_{1}-\lambda_{1} d \lambda_{2}$ [2, Example 13.83]. Its kernel $\mathcal{D}_{Z}$ is isomorphic to $\mathfrak{t}^{1,0} \otimes \mathcal{O}(1)$. Applying Lemma 2 to $\ell=1$, we have

Lemma 3 There is a natural isomorphism $H^{k}\left(Z, \mathcal{D}_{Z}\right)=\mathfrak{t}^{1,0} \otimes \mathfrak{t}^{*(0, k)} \otimes S^{k+1} \mathbf{C}^{2}$. 
We seek local as well as global representations of elements in these cohomology spaces. On $U_{2}$ the product coordinates $\left\{z_{1}^{a}, z_{2}^{a}, \mu\right\}$ are not holomorphic. The holomorphic coordinates are

$$
w_{1}^{a}=\mu z_{1}^{a}-\bar{z}_{2}^{a}, \quad w_{2}^{a}=\mu z_{2}^{a}+\bar{z}_{1}^{a}, \quad \zeta=\mu
$$

The inverse coordinate change is

$$
z_{1}^{a}=\frac{1}{1+|\zeta|^{2}}\left(\bar{\zeta} w_{1}^{a}+\bar{w}_{2}^{a}\right), \quad z_{2}^{a}=\frac{1}{1+|\zeta|^{2}}\left(-\bar{w}_{1}^{a}+\bar{\zeta} w_{2}^{a}\right), \quad \mu=\zeta
$$

When one changes coordinates from $\lambda_{2} \neq 0$ to $\lambda_{1} \neq 0, \mu \tilde{w}_{j}^{a}=w_{j}^{a}$. Therefore,

$$
V_{j}^{a}=\frac{1}{\lambda_{2}} \frac{\partial}{\partial w_{j}^{a}}=\frac{1}{\lambda_{1}} \frac{\partial}{\partial \tilde{w}_{j}^{a}}
$$

is a globally defined section of the tangent bundle.

We are now able to describe a basis for the space $H^{0}\left(Z, \mathcal{D}_{Z}\right)$. The dual of the $(1,0)$-form $\omega_{k}^{a}$ in (17) is

$$
W_{k}^{a}=\frac{1}{2}\left(I_{k} \frac{\partial}{\partial x_{2 a-1}}-i I_{\vec{a}} I_{k} \frac{\partial}{\partial x_{2 a-1}}\right) .
$$

These vector fields can also be identified as

$$
\begin{array}{ll}
W_{0}^{a}=\lambda_{1} V_{1}^{a}+\lambda_{2} V_{2}^{a}, & W_{1}^{a}=i\left(\lambda_{1} V_{1}^{a}-\lambda_{2} V_{2}^{a}\right), \\
W_{2}^{a}=\lambda_{1} V_{2}^{a}-\lambda_{2} V_{1}^{a}, & W_{3}^{a}=i\left(\lambda_{1} V_{2}^{a}+\lambda_{2} V_{1}^{a}\right) .
\end{array}
$$

It follows that the elements $\lambda_{1}^{1-\ell} \lambda_{2}^{\ell} V_{i}^{a} \otimes \Omega_{j}^{b}$, with $0 \leq \ell \leq 1,1 \leq i, j \leq 2,1 \leq a, b \leq m$ form a basis for $H^{1}\left(Z, \mathcal{D}_{Z}\right)$. Similarly, the twisted vector-valued k-forms

$$
\lambda_{1}^{k+1-\ell} \lambda_{2}^{\ell} V_{i}^{a} \otimes \bar{\Omega}_{1}^{a_{1}} \wedge \cdots \wedge \bar{\Omega}_{1}^{a_{i}} \wedge \bar{\Omega}_{2}^{b_{1}} \wedge \cdots \wedge \bar{\Omega}_{2}^{b_{j}}
$$

with $0 \leq \ell \leq k+1$ form a basis for $H^{k}\left(Z, \mathcal{D}_{Z}\right)$.

\subsection{The Twistor space of $X=\check{H} \times T^{3}$}

From the definition of the complex structure on twistor spaces, the projection from $X$ to $T^{4 m}$ induces a natural holomorphic projection $\Psi$ from the twistor space $W$ of $X$ onto the twistor space $Z$ of $T^{4 m}$. Moreover, $p \circ \Psi=p$. We make use of these projections and the related spectral sequences to calculate cohomology on the twistor space $W$. 
Lemma 4 For all $\ell \geq-1, H^{k}\left(W, p^{*} \mathcal{O}(\ell)\right)=\left(\mathfrak{h}_{4 m+1} \oplus \mathfrak{t}_{3}\right)^{*(0, k)} \otimes S^{k+\ell} \mathbf{C}^{2}$.

Proof: The fibers of the projection $p$ from $W$ to $\mathbf{C P}^{1}$ are isomorphic to $\check{H} \times \mathrm{U}(1) \times T^{2}$ where $\check{H} \times U(1)$ is a complex $2 m+1$-dimensional manifold [6 and $T^{2}$ is a complex elliptic curve whose fundamental domain is a square. Since $H^{1}\left(T^{2}, \mathcal{O}\right)$ is one-dimensional, we combine [6, Lemma 2] with Kunneth formula to find that

$$
H^{k}\left(p^{-1}(\vec{a}), \mathcal{O}\right)=\wedge^{k} H^{1}\left(p^{-1}(\vec{a}), \mathcal{O}\right) .
$$

Let $\gamma$ be the dual of the central vector field $Z$ on the Heisenberg group. Denote the dual of $E_{1}, E_{2}$, and $E_{3}$ by $\delta_{1}, \delta_{2}$ and $\delta_{3}$. As in (17), define $\bar{\omega}_{k}^{m+1}=I_{k} \gamma-i I_{\vec{a}} I_{k} \gamma$. Given the stereographic projection (10), we deduce that when $\lambda_{2} \neq 0$,

$$
\begin{array}{ll}
\bar{\omega}_{0}^{m+1}=\mu \bar{\sigma}_{1}^{m+1}+\bar{\sigma}_{2}^{m+1}, & \bar{\omega}_{1}^{m+1}=i\left(\mu \bar{\sigma}_{1}^{m+1}-\bar{\sigma}_{2}^{m+1}\right), \\
\bar{\omega}_{2}^{m+1}=\mu \bar{\sigma}_{2}^{m+1}-\bar{\sigma}_{1}^{m+1}, & \bar{\varpi}_{3}^{m+1}=i\left(\bar{\sigma}_{1}^{m+1}+\mu \bar{\sigma}_{2}^{m+1}\right),
\end{array}
$$

where

$$
\bar{\sigma}_{1}^{m+1}=\frac{\bar{\mu}\left(\gamma-i \delta_{1}\right)-\left(\delta_{2}+i \delta_{3}\right)}{1+|\mu|^{2}}, \quad \bar{\sigma}_{2}^{m+1}=\frac{\left(\gamma+i \delta_{1}\right)+\bar{\mu}\left(\delta_{2}-i \delta_{3}\right)}{1+|\mu|^{2}} .
$$

It is important to note that $d \gamma$ is type $(1,1)$ with respect to any complex structure in the given hypercomplex structure. In fact, the structural equation yields

$$
d \gamma=4 \sum_{a=1}^{m}\left(d x_{2 a-1} \wedge d y_{2 a-1}+d x_{2 a} \wedge d y_{2 a}\right) .
$$

It follows that

$$
\begin{aligned}
d \bar{\sigma}_{1}^{m+1} & =2 \bar{\mu} \sum_{a=1}^{m}\left(\sigma_{1}^{a} \wedge \bar{\sigma}_{2}^{a}+\bar{\sigma}_{1}^{a} \wedge \sigma_{2}^{a}\right)+\frac{d \bar{\mu} \wedge \sigma_{2}^{m+1}-\bar{\mu} d \mu \wedge \bar{\sigma}_{1}^{m+1}}{1+|\mu|^{2}} \\
d \bar{\sigma}_{2}^{m+1} & =2 \sum_{a=1}^{m}\left(\sigma_{1}^{a} \wedge \bar{\sigma}_{2}^{a}+\bar{\sigma}_{1}^{a} \wedge \sigma_{2}^{a}\right)+\frac{-\bar{\mu} d \mu \wedge \bar{\sigma}_{2}^{m+1}-d \bar{\mu} \wedge \sigma_{1}^{m+1}}{1+|\mu|^{2}}
\end{aligned}
$$

In particular, $\bar{\sigma}_{1}^{m+1}$ and $\bar{\sigma}_{2}^{m+1}$ are holomorphic. It shows that the $(0,1)$-forms $\bar{\omega}_{k}^{m+1}$ are holomorphic on the twistor space $W$. These holomorphic $(0,1)$-forms are the analogies of $\bar{\sigma}_{1}^{a}$ and $\bar{\sigma}_{2}^{a}$ on the twistor space $Z$ defined in (13). These 1 -forms on $Z$ are pulled back by $\Psi$ to holomorphic $(0,1)$-forms on $W$. Therefore, $\bar{\sigma}_{i}^{\alpha}$, with $i=1,2$ and $1 \leq \alpha \leq m+1$ form a basis for $H^{1}\left(p^{-1}(\vec{a}), \mathcal{O}\right)$ when $\lambda_{2} \neq 0$. Due to the homogeneity of these forms,

$$
R^{q} p_{*} \mathcal{O}_{W}=\left(\mathfrak{h}_{4 m+1} \oplus \mathfrak{t}_{3}\right)^{*(0, q)} \otimes \mathcal{O}(q) .
$$


Consider the Leray spectral sequence $E_{*}^{p, q}\left(p^{*} \mathcal{O}(\ell)\right)$. Due to the projection formula,

$$
\begin{aligned}
& E_{2}^{p, q}\left(p^{*} \mathcal{O}(\ell)\right)=H^{p}\left(\mathbf{C P}^{1}, R^{q} p_{*} p^{*} \mathcal{O}(\ell)\right)=H^{p}\left(\mathbf{C P}^{1}, \mathcal{O}(\ell) \otimes R^{q} p_{*} \mathcal{O}_{W}\right) \\
= & \left(\mathfrak{h}_{4 m+1} \oplus \mathfrak{t}_{3}\right)^{*(0, q)} \otimes H^{p}\left(\mathbf{C P}^{1}, \mathcal{O}(q+\ell)\right) .
\end{aligned}
$$

As every element in this space is represented by global holomorphic forms on the twistor space $W$ with coefficients in $p^{*} \mathcal{O}(\ell), d_{2}=0$. It follows that for $\ell \geq-1$,

$$
\begin{aligned}
H^{k}\left(W, p^{*} \mathcal{O}(\ell)\right) & =\oplus_{p+q=k} E_{2}^{p, q}\left(p^{*} \mathcal{O}(\ell)\right)=E_{2}^{0, k}\left(p^{*} \mathcal{O}(\ell)\right) \\
& =\left(\mathfrak{h}_{4 m+1} \oplus \mathfrak{t}_{3}\right)^{*(0, k)} \otimes S^{k+\ell} \mathbf{C}^{2}
\end{aligned}
$$

q. e. d.

Since the kernel $\mathcal{V}$ of the differential $d \Psi$ restricted to $\mathcal{D}_{W}$ satisfies $\mathcal{V}=\left(\mathfrak{c} \oplus \mathfrak{t}_{3}\right)^{1,0} \otimes$ $\mathcal{O}(1)$, and since $\Psi^{*} \mathcal{D}_{Z}=\mathfrak{t}_{4 m}^{1,0} \otimes \mathcal{O}(1)$, the next lemma follows.

Lemma 5 Let $\mathcal{V}$ be the kernel of the differential $d \Psi$ restricted to $\mathcal{D}_{W}$. Then

$$
\begin{aligned}
H^{k}(W, \mathcal{V}) & =\left(\mathfrak{c} \oplus \mathfrak{t}_{3}\right)^{1,0} \otimes\left(\mathfrak{h}_{4 m+1} \oplus \mathfrak{t}_{3}\right)^{*(0, k)} \otimes S^{k+1} \mathbf{C}^{2}, \\
H^{k}\left(W, \Psi^{*} \mathcal{D}_{Z}\right) & =\mathfrak{t}_{4 m}^{1,0} \otimes\left(\mathfrak{h}_{4 m+1} \oplus \mathfrak{t}_{3}\right)^{*(0, k)} \otimes S^{k+1} \mathbf{C}^{2} .
\end{aligned}
$$

This lemma implies that $H^{0}(W, \mathcal{V})=\left(\mathfrak{c} \oplus \mathfrak{t}_{3}\right)^{1,0} \otimes \mathbf{C}^{2}$. This space is linearly spanned by the infinitesimal hypercomplex transformations generated by the center of the algebra $\mathfrak{h}_{4 m+1} \oplus \mathfrak{t}_{3}$. It is a linear span of $W_{k}:=\frac{1}{2}\left(I_{k} Z-i I_{\vec{a}} I_{k} Z\right)$.

On $W$, a local holomorphic frame for the bundle of $(0,1)$-forms consists of $\left\{\bar{\sigma}_{i}^{\alpha}, d \bar{\mu}\right\}$. The dual smooth $(0,1)$-vectors are $\partial / \partial \bar{\mu}$ and

$$
\begin{aligned}
\bar{\partial}_{1}^{a} & =\frac{1}{2}\left\{\mu\left(X_{2 a-1}+i X_{2 a}\right)-\left(Y_{2 a-1}-i Y_{2 a}\right)\right\} \\
\bar{\partial}_{2}^{a} & =\frac{1}{2}\left\{\left(X_{2 a-1}-i X_{2 a}\right)+\mu\left(Y_{2 a-1}+i Y_{2 a}\right)\right\} \\
\bar{\partial}_{1}^{m+1} & =\frac{1}{2}\left\{\mu\left(Z+i E_{1}\right)-\left(E_{2}-i E_{3}\right)\right\}, \bar{\partial}_{2}^{m+1}=\frac{1}{2}\left\{\left(Z-i E_{1}\right)+\mu\left(E_{2}+i E_{3}\right)\right\}
\end{aligned}
$$

Now we examine the induced long exact sequence of

$$
0 \rightarrow \mathcal{V} \rightarrow \mathcal{D}_{W} \rightarrow \Psi^{*} \mathcal{D}_{Z} \rightarrow 0
$$

to calculate the cohomology of $\mathcal{D}_{W}$. Note that elements in $H^{0}\left(W, \Psi^{*} \mathcal{D}_{Z}\right)$ are linear combinations of the vector fields (22). They have natural lifting to smooth sections of 
the tangent bundle on $W$, namely $\tilde{W}_{a}^{k}=\frac{1}{2}\left(I_{k} X_{2 a-1}-i I_{\vec{a}} I_{k} X_{2 a-1}\right)$. Given the smooth local frame above,

$$
\begin{array}{ll}
\tilde{W}_{0}^{a}=\frac{1}{1+|\mu|^{2}}\left(\mu \partial_{1}^{a}+\partial_{2}^{a}\right), & \tilde{W}_{1}^{a}=\frac{i}{1+|\mu|^{2}}\left(\mu \partial_{1}^{a}-\partial_{2}^{a}\right), \\
\tilde{W}_{2}^{a}=\frac{1}{1+|\mu|^{2}}\left(\mu \partial_{2}^{a}-\partial_{1}^{a}\right), & \tilde{W}_{3}^{a}=\frac{i}{1+|\mu|^{2}}\left(\mu \partial_{2}^{a}+\partial_{1}^{a}\right) .
\end{array}
$$

Given the algebra structure of the Heisenberg algebra,

$$
\left[\bar{\partial}_{i}^{a}, \partial_{i}^{b}\right]=0,\left[\bar{\partial}_{2}^{a}, \partial_{1}^{b}\right]=-\left[\bar{\partial}_{1}^{a}, \partial_{2}^{b}\right]=2 \delta_{a b}\left(1+|\mu|^{2}\right) Z . \quad\left[\partial_{i}^{a}, \partial_{j}^{b}\right]=0 .
$$

In particular,

$$
\left[\bar{\partial}_{2}^{a}, \partial_{1}^{a}\right]^{1,0}=-\left[\bar{\partial}_{1}^{a}, \partial_{2}^{a}\right]^{1,0}=2\left(1+|\mu|^{2}\right) W_{0} .
$$

A computation using (33) and (36) shows that

$$
\begin{aligned}
{\left[\frac{\partial}{\partial \bar{\mu}}, \tilde{W}_{0}^{a}\right] } & =\frac{1}{\left(1+|\mu|^{2}\right)^{2}}\left(-\bar{\partial}_{1}^{a}+\mu \bar{\partial}_{2}^{a}\right), & {\left[\frac{\partial}{\partial \bar{\mu}}, \tilde{W}_{1}^{a}\right] } & =\frac{i}{\left(1+|\mu|^{2}\right)^{2}}\left(\bar{\partial}_{1}^{a}+\mu \bar{\partial}_{2}^{a}\right),(39) \\
{\left[\frac{\partial}{\partial \bar{\mu}}, \tilde{W}_{2}^{a}\right] } & =\frac{-1}{\left(1+|\mu|^{2}\right)^{2}}\left(\mu \bar{\partial}_{1}^{a}+\bar{\partial}_{2}^{a}\right), & {\left[\frac{\partial}{\partial \bar{\mu}}, \tilde{W}_{3}^{a}\right] } & =\frac{i}{\left(1+|\mu|^{2}\right)^{2}}\left(-\mu \bar{\partial}_{1}^{a}+\bar{\partial}_{2}^{a}\right)(40)
\end{aligned}
$$

In particular, $\left[\frac{\partial}{\partial \bar{\mu}}, \tilde{W}_{k}^{a}\right]^{1,0}=0$ for all $a$ and $k$. Note also that $\left[\bar{\partial}_{j}^{m+1}, \tilde{W}_{k}^{a}\right]=0$ because $Z, E_{1}, E_{2}, E_{3}$ are in the center of $\mathfrak{h}_{4 m+1} \oplus \mathfrak{t}_{3}$. It follows that the 0 -th coboundary map for the induced exact sequence of (35) can be calculated as follows. We use the Chern connection $\nabla$ on the holomorphic tangent bundle on $W$ to define the $\bar{\partial}$ operator $\bar{\partial}^{\nabla}$. Then $\delta_{0}\left(W_{k}^{a}\right)$ is represented uniquely by the cohomology class of $\bar{\partial}^{\nabla} \tilde{W}_{k}^{a}$. Due to an observation of Gauduchon [4], for any $(0,1)$-vector $X$, and $(1,0)$-vector $Y$, $\bar{\partial}_{X}^{\nabla} Y=[X, Y]^{1,0}$. The above discussion implies that

$$
\delta_{0}\left(W_{k}^{a}\right)=\left[\bar{\partial}_{j}^{a}, \tilde{W}_{k}^{a}\right]^{1,0} \otimes \bar{\sigma}_{j}^{a}=\left[\bar{\partial}_{1}^{a}, \tilde{W}_{k}^{a}\right]^{1,0} \otimes \bar{\sigma}_{1}^{a}+\left[\bar{\partial}_{2}^{a}, \tilde{W}_{k}^{a}\right]^{1,0} \otimes \bar{\sigma}_{2}^{a} .
$$

With (36), (37) and (38), we deduce that

$$
\begin{aligned}
& \delta_{0}\left(W_{0}^{a}\right)=2 W_{0} \otimes\left(\lambda_{1} \bar{\Omega}_{2}^{a}-\lambda_{2} \bar{\Omega}_{1}^{a}\right), \delta_{0}\left(W_{1}^{a}\right)=2 i W_{0} \otimes\left(\lambda_{1} \bar{\Omega}_{2}^{a}+\lambda_{2} \bar{\Omega}_{1}^{a}\right), \\
& \delta_{0}\left(W_{2}^{a}\right)=-2 W_{0} \otimes\left(\lambda_{1} \bar{\Omega}_{1}^{a}+\lambda_{2} \bar{\Omega}_{2}^{a}\right), \delta_{0}\left(W_{3}^{a}\right)=-2 i W_{0} \otimes\left(\lambda_{1} \bar{\Omega}_{1}^{a}-\lambda_{2} \bar{\Omega}_{2}^{a}\right) .
\end{aligned}
$$

Therefore, the coboundary map $\delta_{0}$ is injective and $H^{0}\left(W, \mathcal{D}_{W}\right)=H^{0}(W, \mathcal{V})$.

The functions $w_{1}^{m+1}=\mu z_{1}-\bar{z}_{2}$ and $w_{2}^{m+1}=\mu z_{2}+\bar{z}_{1}$ are holomorphic on the open subset $U_{2}$ of the twistor space $W$. On $U_{1}$, define $\tilde{w}_{j}^{m+1}=\frac{1}{\mu} w_{j}^{m+1}$. As in (21) we define $V_{j}:=V_{j}^{m+1}:=\frac{1}{\lambda_{2}} \frac{\partial}{\partial w_{j}^{m+1}}=\frac{1}{\lambda_{1}} \frac{\partial}{\partial \tilde{w}_{j}^{m+1}}$. In this basis, the image of $H^{0}\left(W, \Psi^{*} \mathcal{D}_{Z}\right)$ in $H^{1}(W, \mathcal{V})$ is spanned by $\lambda_{i}\left(\lambda_{1} V_{1}+\lambda_{2} V_{2}\right) \otimes \bar{\Omega}_{j}^{a}$. By Lemma 5 ,

$$
H^{1}(W, \mathcal{V})=\left(\mathfrak{c} \oplus \mathfrak{t}_{3}\right)^{1,0} \otimes\left(\mathfrak{c} \oplus \mathfrak{t}_{3}\right)^{*(0,1)} \otimes S^{2} \mathbf{C}^{2} \oplus\left(\mathfrak{c} \oplus \mathfrak{t}_{3}\right)^{1,0} \otimes \mathfrak{t}_{4 m}^{*(0,1)} \otimes S^{2} \mathbf{C}^{2}
$$


Since the image of $\delta_{0}$ is contained in the second summand, the cokernel of $\delta_{0}$ is the direct sum of the following spaces.

$$
\begin{aligned}
\operatorname{coker}_{0}{ }^{\prime \prime}: & =\left(\mathfrak{c} \oplus \mathfrak{t}_{3}\right)^{1,0} \otimes\left(\mathfrak{c} \oplus \mathfrak{t}_{3}\right)^{*(0,1)} \otimes S^{2} \mathbf{C}^{2}=\operatorname{span}\left\{\lambda_{1}^{k} \lambda_{2}^{2-k} V_{i} \bar{\Omega}_{j}^{m+1}\right\} \\
\operatorname{coker}_{0}{ }^{\prime}: & =\operatorname{span}\left\{\left(\lambda_{1} V_{1}-\lambda_{2} V_{2}\right) \lambda_{i} \bar{\Omega}_{j}^{a}, \lambda_{2}^{2} V_{1} \bar{\Omega}_{j}^{a}, \lambda_{1}^{2} V_{2} \bar{\Omega}_{j}^{a}\right\}
\end{aligned}
$$

where $\bar{\Omega}_{j}^{m+1}=\frac{1}{\lambda_{2}} \bar{\sigma}_{j}^{m+1}$.

A basis for $H^{1}\left(W, \Psi^{*} \mathcal{D}_{Z}\right)$ consists of $\lambda_{1}^{2-k} \lambda_{2}^{k} V_{i}^{a} \bar{\Omega}_{j}^{\beta}$, where $0 \leq k \leq 2,1 \leq i, j \leq 2$,

$1 \leq a \leq m, 1 \leq \beta \leq m+1$. Since $\bar{\Omega}_{j}^{\beta}$ is holomorphic, the coboundary map $\delta_{1}$ from the first cohomology to the second has the following property.

$$
\delta_{1}\left(V_{1}^{a} \bar{\Omega}_{j}^{\beta}\right)=2\left(\lambda_{1} V_{1}+\lambda_{2} V_{2}\right) \otimes \bar{\Omega}_{2}^{a} \wedge \bar{\Omega}_{j}^{\beta}, \delta_{1}\left(V_{2}^{a} \bar{\Omega}_{j}^{\beta}\right)=-2\left(\lambda_{1} V_{1}+\lambda_{2} V_{2}\right) \otimes \bar{\Omega}_{1}^{a} \wedge \bar{\Omega}_{j}^{\beta} .
$$

Therefore, the kernel of $\delta_{1}$ is

$$
\operatorname{ker} \delta_{1}=\operatorname{span}\left\{\lambda_{1}^{k} \lambda_{2}^{2-k}\right\} \otimes \operatorname{span}\left\{V_{1}^{a} \bar{\Omega}_{2}^{b}+V_{1}^{b} \bar{\Omega}_{2}^{a}, V_{2}^{a} \bar{\Omega}_{1}^{b}+V_{2}^{b} \bar{\Omega}_{1}^{a}, V_{1}^{a} \bar{\Omega}_{1}^{b}-V_{2}^{b} \bar{\Omega}_{2}^{a}\right\} .
$$

The induced exact sequence yields

$$
\begin{aligned}
H^{1}\left(W, \mathcal{D}_{W}\right) & =\text { coker }_{0}^{\prime \prime} \oplus \operatorname{coker} \delta_{0}^{\prime} \oplus \operatorname{ker} \delta_{1} \\
& =\left\{\lambda_{1}^{k} \lambda_{2}^{2-k} V_{i} \bar{\Omega}_{j}^{m+1}\right\} \oplus\left\{\left(\lambda_{1} V_{1}-\lambda_{2} V_{2}\right) \lambda_{i} \bar{\Omega}_{j}^{a}, \lambda_{2}^{2} V_{1} \bar{\Omega}_{j}^{a}, \lambda_{1}^{2} V_{2} \bar{\Omega}_{j}^{a}\right\} \\
& \oplus\left\{\lambda_{1}^{k} \lambda_{2}^{2-k}\right\} \otimes\left\{V_{1}^{a} \bar{\Omega}_{2}^{b}+V_{1}^{b} \bar{\Omega}_{2}^{a}, V_{2}^{a} \bar{\Omega}_{1}^{b}+V_{2}^{b} \bar{\Omega}_{1}^{a}, V_{1}^{a} \bar{\Omega}_{1}^{b}-V_{2}^{b} \bar{\Omega}_{2}^{a}\right\}
\end{aligned}
$$

Here we see that $\operatorname{coker} \delta_{0}{ }^{\prime \prime}$ is a 12 -dimensional space. It is generated by vertical tangent vectors and 1 -forms with respect to the projection $\Psi$. The dimension of $\operatorname{coker} \delta_{0}{ }^{\prime}$ is equal to $8 \mathrm{~m}$. The contribution from deformation of the basis of the projection $\Psi$ is in $k e r \delta_{1}$. The dimension of this space is equal to $3 m(2 m+1)$. This concludes the proof of Theorem [1.

\section{Deformation Theory}

The obstruction space to hypercomplex deformation is $H^{2}\left(W, \mathcal{D}_{W}\right)$. Results in the last section demonstrate that it does not vanish, we approach integrability of parameters in $H^{1}\left(W, \mathcal{D}_{W}\right)$ by constructing a one-parameter deformation for any given tangent. It is done through Kodaira-Spencer's method of constructing a convergent sequence with coefficients in vector-valued 1-forms. During this construction, we do not control the vector-valued 1-forms in such a way that the vector part is tangent to the distribution $\mathcal{D}_{W}$. Therefore, the deformation may a priori depart from hypercomplex structures. We tackle this problem at the end of this section. 


\subsection{Integrability}

We have seen that the virtual parameter space $H^{1}(W, \mathcal{D})$ is a vector subspace of the following space.

$$
\begin{aligned}
\mathcal{E} & =H^{1}(W, \mathcal{V}) \oplus \operatorname{ker} \delta_{1} \\
& =\left(\mathfrak{c} \oplus \mathfrak{t}_{3}\right)^{1,0} \otimes\left(\mathfrak{h}_{4 m+1} \oplus \mathfrak{t}_{3}\right)^{*(0,1)} \otimes S^{2} \mathbf{C}^{2} \oplus \operatorname{ker} \delta_{1} \\
& =\left\langle\lambda_{1}^{k} \lambda_{2}^{2-k}\right\rangle \otimes\left(\left\langle V_{i}^{m+1} \bar{\Omega}_{j}^{\beta}\right\rangle \oplus\left\langle V_{1}^{a} \bar{\Omega}_{2}^{b}+V_{1}^{b} \bar{\Omega}_{2}^{a}, V_{2}^{a} \bar{\Omega}_{1}^{b}+V_{2}^{b} \bar{\Omega}_{1}^{a}, V_{1}^{a} \bar{\Omega}_{1}^{b}-V_{2}^{b} \bar{\Omega}_{2}^{a}\right\rangle\right)(4
\end{aligned}
$$

We denote these elements by $\Upsilon_{\tau}$, where $1 \leq \tau \leq D=\operatorname{dim} \mathcal{E}$. If $\Omega_{1}=\omega \otimes V$ and $\Omega_{2}=\omega^{\prime} \otimes V^{\prime}$ are vector-valued 1-forms, the Dolbeault representative for the Nijenhuis bracket $\left\{\omega \otimes V, \omega^{\prime} \otimes V^{\prime}\right\}$ is [14, 5]

$$
\omega^{\prime} \wedge L_{V^{\prime}} \omega \otimes V+\omega \wedge L_{V} \omega^{\prime} \otimes V^{\prime}+\omega \wedge \omega^{\prime} \otimes\left[V, V^{\prime}\right]
$$

The aim of this section is to prove the following proposition.

Proposition 1 Let $\Gamma^{0}$ be the complex vector space of complex-valued smooth functions on $S^{2}$. For any $\phi_{1}$ and $\phi_{2}$ in $\Gamma^{0} \otimes \mathcal{E}$, there exist smooth functions $g_{\tau}$ on the 2-sphere such that $\left\{\phi_{1}, \phi_{2}\right\}=\sum_{\tau} \bar{\partial}\left(g_{\tau} \Upsilon_{\tau}\right)$.

The following functions on 2-sphere $S^{2}$ is extended to the twistor space $W$.

$$
f_{1}=\frac{\mu}{1+|\mu|^{2}}, \quad f_{2}=\frac{\bar{\mu}}{1+|\mu|^{2}}, \quad f_{3}=\frac{1}{1+|\mu|^{2}} .
$$

We have

$$
\bar{\partial} f_{1}=-\frac{\mu^{2} d \bar{\mu}}{\left(1+|\mu|^{2}\right)^{2}}, \quad \bar{\partial} f_{2}=\frac{d \bar{\mu}}{\left(1+|\mu|^{2}\right)^{2}}, \quad \bar{\partial} f_{3}=-\frac{\mu d \bar{\mu}}{\left(1+|\mu|^{2}\right)^{2}} .
$$

In subsequent computation, we make use of these three functions to prove the Proposition 1 .

Let $\epsilon_{11}=\epsilon_{22}=0$ and $\epsilon_{12}=-\epsilon_{21}=1$. It follows from (33) and (34) that

$$
\left[\partial_{i}^{\alpha}, \frac{\partial}{\partial \bar{\mu}}\right]^{0,1}=-\frac{\epsilon_{i j}}{1+|\mu|^{2}} \partial_{j}^{\alpha}, \text { and } L_{\partial_{i}^{\alpha}} \bar{\sigma}_{j}^{\beta}=\delta_{\alpha \beta} \epsilon_{i j} \frac{d \bar{\mu}}{1+|\mu|^{2}}
$$

except when $\alpha=a, \beta=m+1$. Therefore, in the case when $\alpha=\alpha^{\prime}=m+1$ or when $1 \leq \beta, \beta^{\prime} \leq m$, with (37) we deduce that

$$
\left\{\lambda_{1}^{k} \lambda_{2}^{2-k} V_{i}^{\alpha} \bar{\Omega}_{j}^{\beta}, \lambda_{1}^{k^{\prime}} \lambda_{2}^{2-k^{\prime}} V_{i^{\prime}}^{\alpha^{\prime}} \bar{\Omega}_{j^{\prime}}^{\beta^{\prime}}\right\}
$$




$$
\begin{aligned}
& =\left\{\frac{\mu^{k}}{1+|\mu|^{2}} \partial_{i}^{\alpha} \bar{\sigma}_{j}^{\beta}, \frac{\mu^{k^{\prime}}}{1+|\mu|^{2}} \partial_{i^{\prime}}^{\alpha^{\prime}} \bar{\sigma}_{j^{\prime}}^{\beta^{\prime}}\right\}=\frac{\mu^{k+k^{\prime}}}{\left(1+|\mu|^{2}\right)^{2}}\left\{\partial_{i}^{\alpha} \bar{\sigma}_{j}^{\beta}, \partial_{i^{\prime}}^{\alpha^{\prime}} \bar{\sigma}_{j^{\prime}}^{\beta^{\prime}}\right\} \\
& =\frac{\mu^{k+k^{\prime}}}{\left(1+|\mu|^{2}\right)^{2}}\left(\partial_{i}^{\alpha} \bar{\sigma}_{j^{\prime}}^{\beta^{\prime}} \wedge L_{\partial_{i^{\prime}}^{\alpha^{\prime}}}\left(\bar{\sigma}_{j}^{\beta}\right)+\partial_{i^{\prime}}^{\alpha^{\prime}} \bar{\sigma}_{j}^{\beta} \wedge L_{\partial_{i}^{\alpha}}\left(\bar{\sigma}_{j^{\prime}}^{\beta^{\prime}}\right)+\left[\partial_{i}^{\alpha}, \partial_{i^{\prime}}^{\alpha^{\prime}}\right] \bar{\sigma}_{j}^{\beta} \wedge \bar{\sigma}_{j^{\prime}}^{\beta^{\prime}}\right) \\
& =\frac{\mu^{k+k^{\prime}}}{\left(1+|\mu|^{2}\right)^{2}}\left(\partial_{i}^{\alpha} \bar{\sigma}_{j^{\prime}}^{\beta^{\prime}} \wedge L_{\partial_{i^{\prime}}^{\alpha^{\prime}}}\left(\bar{\sigma}_{j}^{\beta}\right)+\partial_{i^{\prime}}^{\alpha^{\prime}} \bar{\sigma}_{j}^{\beta} \wedge L_{\partial_{i}^{\alpha}}\left(\bar{\sigma}_{j^{\prime}}^{\beta^{\prime}}\right)\right) \\
& =-\frac{\mu^{k+k^{\prime}}}{\left(1+|\mu|^{2}\right)^{3}} d \bar{\mu} \wedge\left(\delta_{\alpha^{\prime} \beta} \epsilon_{i^{\prime} j} \partial_{i}^{\alpha} \bar{\sigma}_{j^{\prime}}^{\beta^{\prime}}+\delta_{\alpha \beta^{\prime}} \epsilon_{i j^{\prime}} \partial_{i^{\prime}}^{\alpha^{\prime}} \bar{\sigma}_{j}^{\beta}\right) \\
& =-\frac{\mu^{k^{\prime}} d \bar{\mu}}{\left(1+|\mu|^{2}\right)^{2}} \wedge\left(\frac{\delta_{\alpha^{\prime} \beta} \epsilon_{i^{\prime} j} \mu^{k}}{1+|\mu|^{2}} \partial_{i}^{\alpha} \bar{\sigma}_{j^{\prime}}^{\beta^{\prime}}\right)-\frac{\mu^{k^{\prime}} d \bar{\mu}}{\left(1+|\mu|^{2}\right)^{2}} \wedge\left(\frac{\delta_{\alpha \beta^{\prime}} \epsilon_{i j^{\prime}} \mu^{k}}{1+|\mu|^{2}} \partial_{i^{\prime}}^{\alpha^{\prime}} \bar{\sigma}_{j}^{\beta}\right) \\
& =\overline{\mu^{k^{\prime}} d \bar{\mu}} \wedge\left(\delta_{\alpha^{\prime} \beta} \epsilon_{i^{\prime} j} \lambda_{1}^{k} \lambda_{2}^{2-k} V_{i}^{\alpha} \bar{\Omega}_{j^{\prime}}^{\beta^{\prime}}\right)-\frac{\mu^{k^{\prime}} d \bar{\mu}}{\left(1+|\mu|^{2}\right)^{2}} \wedge\left(\delta_{\alpha \beta^{\prime}} \epsilon_{i j^{\prime}} \lambda_{1}^{k} \lambda_{2}^{2-k} V_{i^{\prime}}^{\alpha^{\prime}} \bar{\Omega}_{j}^{\beta}\right) \\
& =\bar{\partial}\left(-f \delta_{\alpha^{\prime} \beta} \epsilon_{i^{\prime} j} \lambda_{1}^{k} \lambda_{2}^{2-k} V_{i}^{\alpha} \bar{\Omega}_{j^{\prime}}^{\beta^{\prime}}-f \delta_{\alpha \beta^{\prime}} \epsilon_{i j^{\prime}} \lambda_{1}^{k} \lambda_{2}^{2-k} V_{i^{\prime}}^{\alpha^{\prime}} \bar{\Omega}_{j}^{\beta}\right) \\
& =\bar{\partial}\left(\left(-f \lambda_{1}^{k} \lambda_{2}^{2-k}\right)\left(\delta_{\alpha^{\prime} \beta} \epsilon_{i^{\prime} j} V_{i}^{\alpha} \bar{\Omega}_{j^{\prime}}^{\beta^{\prime}}+\delta_{\alpha \beta^{\prime}} \epsilon_{i j^{\prime}} V_{i^{\prime}}^{\alpha^{\prime}} \bar{\Omega}_{j}^{\beta}\right)\right) .
\end{aligned}
$$

where $f$ is determined by $k^{\prime}$.

Lemma 6 For any $\phi_{1}$ and $\phi_{2}$ in $H^{1}(W, \mathcal{V})$, there exist smooth functions $g_{\tau}$ on the 2-sphere and $\phi_{\tau} \in H^{1}(W, \mathcal{V})$ such that $\left\{\phi_{1}, \phi_{2}\right\}=\sum_{\tau} \bar{\partial}\left(g_{\tau} \phi_{\tau}\right)$.

Proof: By Lemma 5, elements in $H^{1}(W, \mathcal{V})$ have the form $\lambda_{1}^{k} \lambda_{2}^{2-k} V_{i}^{m+1} \bar{\Omega}_{j}^{\beta}$ for some $k, j$ and $\beta$. From the above computation,

$$
\begin{aligned}
& \left\{\lambda_{1}^{k} \lambda_{2}^{2-k} V_{i}^{m+1} \bar{\Omega}_{j}^{\beta}, \lambda_{1}^{k^{\prime}} \lambda_{2}^{2-k^{\prime}} V_{i^{\prime}}^{m+1} \bar{\Omega}_{j^{\prime}}^{\beta^{\prime}}\right\} \\
= & \bar{\partial}\left(\left(-f \lambda_{1}^{k} \lambda_{2}^{2-k}\right)\left(\delta_{m+1, \beta} \epsilon_{i^{\prime} j} V_{i}^{m+1} \bar{\Omega}_{j^{\prime}}^{\beta^{\prime}}+\delta_{m+1, \beta^{\prime}} \epsilon_{i j^{\prime}} V_{i^{\prime}}^{m+1} \bar{\Omega}_{j}^{\beta}\right)\right) .
\end{aligned}
$$

This is an element in the image of $\Gamma^{0} \otimes H^{1}(W, \mathcal{V})$ via $\bar{\partial}$. q. e. d.

Lemma 7 For any $\phi_{1}$ and $\phi_{2}$ in ker $\delta_{1}$, there exist smooth functions $g_{\tau}$ on the 2sphere and $\phi_{\tau} \in \operatorname{ker} \delta_{1}$ such that $\left\{\phi_{1}, \phi_{2}\right\}=\sum_{\tau} \bar{\partial}\left(g_{\tau} \phi_{\tau}\right)$.

Proof: We do it through a case-by-case computation. In view of formula (42), there are six cases.

$$
\begin{aligned}
& \left\{\lambda_{1}^{k} \lambda_{2}^{2-k}\left(V_{1}^{a} \bar{\Omega}_{2}^{b}+V_{1}^{b} \bar{\Omega}_{2}^{a}\right), \lambda_{1}^{k^{\prime}} \lambda_{2}^{2-k^{\prime}}\left(V_{1}^{a^{\prime}} \bar{\Omega}_{2}^{b^{\prime}}+V_{1}^{b^{\prime}} \bar{\Omega}_{2}^{a^{\prime}}\right)\right\} \\
= & \bar{\partial}\left(-f \lambda_{1}^{k} \lambda_{2}^{2-k}\left(\delta_{a^{\prime} b} V_{1}^{a} \bar{\Omega}_{2}^{b^{\prime}}+\delta_{a b^{\prime}} V_{1}^{a^{\prime}} \bar{\Omega}_{2}^{b}\right)\right)+\bar{\partial}\left(-f \lambda_{1}^{k} \lambda_{2}^{2-k}\left(\delta_{b^{\prime} b} V_{1}^{a} \bar{\Omega}_{2}^{a^{\prime}}+\delta_{a a^{\prime}} V_{1}^{b^{\prime}} \bar{\Omega}_{2}^{b}\right)\right)
\end{aligned}
$$




$$
\begin{aligned}
& +\bar{\partial}\left(-f \lambda_{1}^{k} \lambda_{2}^{2-k}\left(\delta_{a^{\prime} a} V_{1}^{b} \bar{\Omega}_{2}^{b^{\prime}}+\delta_{b b^{\prime}} V_{1}^{a^{\prime}} \bar{\Omega}_{2}^{a}\right)\right)+\bar{\partial}\left(-f \lambda_{1}^{k} \lambda_{2}^{2-k}\left(\delta_{b^{\prime} a} V_{1}^{b} \bar{\Omega}_{2}^{a^{\prime}}+\delta_{b a^{\prime}} V_{1}^{b^{\prime}} \bar{\Omega}_{2}^{a}\right)\right) \\
= & \bar{\partial}\left(-f \lambda_{1}^{k} \lambda_{2}^{2-k} \delta_{a^{\prime} b}\left(V_{1}^{a} \bar{\Omega}_{2}^{b^{\prime}}+V_{1}^{b^{\prime}} \bar{\Omega}_{2}^{a}\right)\right)+\bar{\partial}\left(-f \lambda_{1}^{k} \lambda_{2}^{2-k} \delta_{a b^{\prime}}\left(V_{1}^{a^{\prime}} \bar{\Omega}_{2}^{b}+V_{1}^{b} \bar{\Omega}_{2}^{a^{\prime}}\right)\right) \\
& +\bar{\partial}\left(-f \lambda_{1}^{k} \lambda_{2}^{2-k} \delta_{b b^{\prime}}\left(V_{1}^{a} \bar{\Omega}_{2}^{a^{\prime}}+V_{1}^{a^{\prime}} \bar{\Omega}_{2}^{a}\right)\right)+\bar{\partial}\left(-f \lambda_{1}^{k} \lambda_{2}^{2-k} \delta_{a a^{\prime}}\left(V_{1}^{b} \bar{\Omega}_{2}^{b^{\prime}}+V_{1}^{b^{\prime}} \bar{\Omega}_{2}^{b}\right)\right) .
\end{aligned}
$$

This element is in the image of $\Gamma^{0} \otimes \operatorname{ker} \delta_{1}$ via $\bar{\partial}$. Similarly, when we interchange the indices 1 and 2, we have

$$
\begin{aligned}
& \left\{\lambda_{1}^{k} \lambda_{2}^{2-k}\left(V_{2}^{a} \bar{\Omega}_{1}^{b}+V_{2}^{b} \bar{\Omega}_{1}^{a}\right), \lambda_{1}^{k^{\prime}} \lambda_{2}^{2-k^{\prime}}\left(V_{2}^{a^{\prime}} \bar{\Omega}_{1}^{b^{\prime}}+V_{2}^{b^{\prime}} \bar{\Omega}_{1}^{a^{\prime}}\right)\right\} \\
= & \bar{\partial}\left(f \lambda_{1}^{k} \lambda_{2}^{2-k} \delta_{a^{\prime} b}\left(V_{2}^{a} \bar{\Omega}_{1}^{b^{\prime}}+V_{2}^{b^{\prime}} \bar{\Omega}_{1}^{a}\right)\right)+\bar{\partial}\left(f \lambda_{1}^{k} \lambda_{2}^{2-k} \delta_{a b^{\prime}}\left(V_{2}^{a^{\prime}} \bar{\Omega}_{1}^{b}+V_{2}^{b} \bar{\Omega}_{1}^{a^{\prime}}\right)\right) \\
& +\bar{\partial}\left(f \lambda_{1}^{k} \lambda_{2}^{2-k} \delta_{b b^{\prime}}\left(V_{2}^{a} \bar{\Omega}_{1}^{a^{\prime}}+V_{2}^{a^{\prime}} \bar{\Omega}_{1}^{a}\right)\right)+\bar{\partial}\left(f \lambda_{1}^{k} \lambda_{2}^{2-k} \delta_{a a^{\prime}}\left(V_{2}^{b} \bar{\Omega}_{1}^{b^{\prime}}+V_{2}^{b^{\prime}} \bar{\Omega}_{1}^{b}\right)\right) .
\end{aligned}
$$

This element is in the image of $\Gamma^{0} \otimes \operatorname{ker} \delta_{1}$ via $\bar{\partial}$. Next,

$$
\begin{aligned}
& \left\{\lambda_{1}^{k} \lambda_{2}^{2-k}\left(V_{1}^{a} \bar{\Omega}_{1}^{b}-V_{2}^{b} \bar{\Omega}_{2}^{a}\right), \lambda_{1}^{k^{\prime}} \lambda_{2}^{2-k^{\prime}}\left(V_{1}^{a^{\prime}} \bar{\Omega}_{1}^{b^{\prime}}-V_{2}^{b^{\prime}} \bar{\Omega}_{2}^{a^{\prime}}\right)\right\} \\
= & -\bar{\partial}\left(f \lambda_{1}^{k} \lambda_{2}^{2-k}\left(-\delta_{b^{\prime} b} V_{1}^{a} \bar{\Omega}_{2}^{a^{\prime}}+\delta_{a a^{\prime}} V_{2}^{b^{\prime}} \bar{\Omega}_{1}^{b}\right)\right)-\bar{\partial}\left(f \lambda_{1}^{k} \lambda_{2}^{2-k}\left(\delta_{a^{\prime} a} V_{2}^{b} \bar{\Omega}_{1}^{b^{\prime}}-\delta_{b b^{\prime}} V_{1}^{a^{\prime}} \bar{\Omega}_{2}^{a}\right)\right) \\
= & \bar{\partial}\left(f \lambda_{1}^{k} \lambda_{2}^{2-k} \delta_{b^{\prime} b}\left(V_{1}^{a} \bar{\Omega}_{2}^{a^{\prime}}+V_{1}^{a^{\prime}} \bar{\Omega}_{2}^{a}\right)\right)-\bar{\partial}\left(f \lambda_{1}^{k} \lambda_{2}^{2-k} \delta_{a^{\prime} a}\left(V_{2}^{b} \bar{\Omega}_{1}^{b^{\prime}}+V_{2}^{b^{\prime}} \bar{\Omega}_{1}^{b}\right)\right)
\end{aligned}
$$

This element is in the image of $\Gamma^{0} \otimes \operatorname{ker} \delta_{1}$ via $\bar{\partial}$. On the other hand,

$$
\begin{aligned}
& \left\{\lambda_{1}^{k} \lambda_{2}^{2-k}\left(V_{1}^{a} \bar{\Omega}_{2}^{b}+V_{1}^{b} \bar{\Omega}_{2}^{a}\right), \lambda_{1}^{k^{\prime}} \lambda_{2}^{2-k^{\prime}}\left(V_{2}^{a^{\prime}} \bar{\Omega}_{1}^{b^{\prime}}+V_{2}^{b^{\prime}} \bar{\Omega}_{1}^{a^{\prime}}\right)\right\}=0 . \\
& \left\{\lambda_{1}^{k} \lambda_{2}^{2-k}\left(V_{1}^{a} \bar{\Omega}_{2}^{b}+V_{1}^{b} \bar{\Omega}_{2}^{a}\right), \lambda_{1}^{k^{\prime}} \lambda_{2}^{2-k^{\prime}}\left(V_{1}^{a^{\prime}} \bar{\Omega}_{1}^{b^{\prime}}-V_{2}^{b^{\prime}} \bar{\Omega}_{2}^{a^{\prime}}\right)\right\} \\
= & \bar{\partial}\left(f \lambda_{1}^{k} \lambda_{2}^{2-k} \delta_{a^{\prime} b} V_{1}^{a} \bar{\Omega}_{1}^{b^{\prime}}\right)-\bar{\partial}\left(f \lambda_{1}^{k} \lambda_{2}^{2-k} \delta_{a a^{\prime}} V_{2}^{b^{\prime}} \bar{\Omega}_{2}^{b}\right) \\
& +\bar{\partial}\left(f \lambda_{1}^{k} \lambda_{2}^{2-k} \delta_{a^{\prime} a} V_{1}^{b} \bar{\Omega}_{1}^{b^{\prime}}\right)-\bar{\partial}\left(f \lambda_{1}^{k} \lambda_{2}^{2-k} \delta_{b a^{\prime}} V_{2}^{b^{\prime}} \bar{\Omega}_{2}^{a}\right) \\
= & \bar{\partial}\left(f \lambda_{1}^{k} \lambda_{2}^{2-k} \delta_{a^{\prime} b}\left(V_{1}^{a} \bar{\Omega}_{1}^{b^{\prime}}-V_{2}^{b^{\prime}} \bar{\Omega}_{2}^{a}\right)\right)+\bar{\partial}\left(f \lambda_{1}^{k} \lambda_{2}^{2-k} \delta_{a^{\prime} a}\left(V_{1}^{b} \bar{\Omega}_{1}^{b^{\prime}}-V_{2}^{b^{\prime}} \bar{\Omega}_{2}^{b}\right)\right) .
\end{aligned}
$$

This element is in the image of $\Gamma^{0} \otimes \operatorname{ker} \delta_{1}$ via $\bar{\partial}$. Finally,

$$
\begin{aligned}
& \left\{\lambda_{1}^{k} \lambda_{2}^{2-k}\left(V_{2}^{a} \bar{\Omega}_{1}^{b}+V_{2}^{b} \bar{\Omega}_{1}^{a}\right), \lambda_{1}^{k^{\prime}} \lambda_{2}^{2-k^{\prime}}\left(V_{1}^{a^{\prime}} \bar{\Omega}_{1}^{b^{\prime}}-V_{2}^{b^{\prime}} \bar{\Omega}_{2}^{a^{\prime}}\right)\right\} \\
= & -\bar{\partial}\left(f \lambda_{1}^{k} \lambda_{2}^{2-k} \delta_{a b^{\prime}} V_{1}^{a^{\prime}} \bar{\Omega}_{1}^{b}\right)+\bar{\partial}\left(f \lambda_{1}^{k} \lambda_{2}^{2-k} \delta_{b^{\prime} b} V_{2}^{a} \bar{\Omega}_{2}^{a^{\prime}}\right) \\
& -\bar{\partial}\left(f \lambda_{1}^{k} \lambda_{2}^{2-k} \delta_{b b^{\prime}} V_{1}^{a^{\prime}} \bar{\Omega}_{1}^{a}\right)+\bar{\partial}\left(f \lambda_{1}^{k} \lambda_{2}^{2-k} \delta_{b^{\prime} a} V_{2}^{b} \bar{\Omega}_{2}^{a^{\prime}}\right) \\
= & -\bar{\partial}\left(f \lambda_{1}^{k} \lambda_{2}^{2-k} \delta_{a b^{\prime}}\left(V_{1}^{a^{\prime}} \bar{\Omega}_{1}^{b}-V_{2}^{b} \bar{\Omega}_{2}^{a^{\prime}}\right)\right)-\bar{\partial}\left(f \lambda_{1}^{k} \lambda_{2}^{2-k} \delta_{b b^{\prime}}\left(V_{1}^{a^{\prime}} \bar{\Omega}_{1}^{a}-V_{2}^{a} \bar{\Omega}_{2}^{a^{\prime}}\right)\right) .
\end{aligned}
$$


This element is in the image of $\Gamma^{0} \otimes \operatorname{ker} \delta_{1}$ via $\bar{\partial}$. q. e. d.

To consider the Nijenhuis bracket between elements in $H^{1}(W, \mathcal{V})$ and elements in $\operatorname{ker} \delta_{1}$, we recall that $H^{1}(W, \mathcal{V})$ has two types of elements: $\lambda_{1}^{k} \lambda_{2}^{2-k} V_{i}^{m+1} \bar{\Omega}_{j}^{m+1}$ as elements in coker $\delta_{0}$ " and $\lambda_{1}^{k} \lambda_{2}^{2-k} V_{i}^{m+1} \bar{\Omega}_{j}^{b}$ as elements in the direct summand complement in $H^{1}(W, \mathcal{V})$. We separate the computation into the next two lemmas. This computation involves the algebraic structure of Heisenberg group because by (29) and (30),

$$
L_{\partial_{1}^{a}} \bar{\sigma}_{1}^{m+1}=2 \overline{\mu \sigma}_{2}^{a}, \quad L_{\partial_{2}^{a}} \bar{\sigma}_{1}^{m+1}=-2 \overline{\mu \sigma}_{1}^{a}, \quad L_{\partial_{1}^{a}} \bar{\sigma}_{2}^{m+1}=2 \bar{\sigma}_{2}^{a}, \quad L_{\partial_{2}^{a}} \bar{\sigma}_{2}^{m+1}=-2 \bar{\sigma}_{1}^{a} .
$$

Lemma 8 For any $\phi_{1}$ in $k e r \delta_{1}$ and $\phi_{2}=\lambda_{1}^{k^{\prime}} \lambda_{2}^{2-k^{\prime}} V_{i^{\prime}}^{m+1} \bar{\Omega}_{j^{\prime}}^{b^{\prime}}$, there exist smooth functions $g_{\tau}$ on the 2-sphere and $\phi_{\tau} \in H^{1}(W, \mathcal{V})$ such that $\left\{\phi_{1}, \phi_{2}\right\}=\sum_{\tau} \bar{\partial}\left(g_{\tau} \phi_{\tau}\right)$.

Proof: We repeat part of a previous computation as follows.

$$
\begin{aligned}
& \left\{\lambda_{1}^{k} \lambda_{2}^{2-k} V_{i}^{a} \bar{\Omega}_{j}^{b}, \lambda_{1}^{k^{\prime}} \lambda_{2}^{2-k^{\prime}} V_{i^{\prime}}^{m+1} \bar{\Omega}_{j^{\prime}}^{b^{\prime}}\right\} \\
= & \frac{\mu^{k+k^{\prime}}}{\left(1+|\mu|^{2}\right)^{2}}\left(\partial_{i}^{a} \bar{\sigma}_{j^{\prime}}^{b^{\prime}} \wedge L_{\partial_{i^{\prime}}^{m+1}}\left(\bar{\sigma}_{j}^{b}\right)+\partial_{i^{\prime}}^{m+1} \bar{\sigma}_{j}^{b} \wedge L_{\partial_{i}^{a}}\left(\bar{\sigma}_{j^{\prime}}^{b^{\prime}}\right)\right) \\
= & \frac{\mu^{k+k^{\prime}}}{\left(1+|\mu|^{2}\right)^{2}}\left(\partial_{i^{\prime}}^{m+1} \bar{\sigma}_{j}^{b} \wedge L_{\partial_{i}^{a}}\left(\bar{\sigma}_{j^{\prime}}^{b^{\prime}}\right)\right)=\frac{\mu^{k+k^{\prime}}}{\left(1+|\mu|^{2}\right)^{2}} \partial_{i^{\prime}}^{m+1} \bar{\sigma}_{j}^{b} \wedge \delta_{a b^{\prime}} \epsilon_{i j^{\prime}} \frac{d \bar{\mu}}{1+|\mu|^{2}} \\
= & -\frac{\mu^{k^{\prime}}}{\left(1+|\mu|^{2}\right)^{2}}\left(\delta_{a b^{\prime}} \epsilon_{i j^{\prime}} \frac{\mu^{k}}{1+|\mu|^{2}} \partial_{i^{\prime}}^{m+1} \bar{\sigma}_{j}^{b}\right)=-\bar{\partial}\left(f \lambda_{1}^{k} \lambda_{2}^{2-k} \delta_{a b^{\prime}} \epsilon_{i j^{\prime}} V_{i^{\prime}}^{m+1} \bar{\Omega}_{j}^{b}\right),
\end{aligned}
$$

where $f$ is one of the functions $f_{1}, f_{2}$ and $f_{3}$ depending on the number $k^{\prime}$. Since $\lambda_{1}^{k} \lambda_{2}^{2-k} V_{i^{\prime}}^{m+1} \bar{\Omega}_{j}^{b}$ is in $H^{1}(W, \mathcal{V})$, the proof of this lemma is completed. q. e. d.

Lemma 9 For any $\phi_{1}$ in $\operatorname{ker} \delta_{1}$ and $\phi_{2}=\lambda_{1}^{k^{\prime}} \lambda_{2}^{2-k^{\prime}} V_{i^{\prime}}^{m+1} \bar{\Omega}_{j^{\prime}}^{m+1},\left\{\phi_{1}, \phi_{2}\right\}=0$.

Proof: We have six cases to consider.

$$
\begin{aligned}
& \left\{\lambda_{1}^{k} \lambda_{2}^{2-k}\left(V_{1}^{a} \bar{\Omega}_{2}^{b}+V_{1}^{b} \bar{\Omega}_{2}^{a}\right), \lambda_{1}^{k^{\prime}} \lambda_{2}^{2-k^{\prime}} V_{i^{\prime}}^{m+1} \bar{\Omega}_{1}^{m+1}\right\} \\
= & \frac{\mu^{k+k^{\prime}} \partial_{i^{\prime}}^{m+1}}{\left(1+|\mu|^{2}\right)^{2}}\left(\bar{\sigma}_{2}^{b} \wedge L_{\partial_{1}^{a}} \bar{\sigma}_{1}^{m+1}+\bar{\sigma}_{2}^{a} \wedge L_{\partial_{1}^{b}} \bar{\sigma}_{1}^{m+1}\right) \\
= & 2 \bar{\mu} \frac{\mu^{k+k^{\prime}}}{\left(1+|\mu|^{2}\right)^{2}} \partial_{i^{\prime}}^{m+1}\left(\bar{\sigma}_{2}^{b} \wedge \bar{\sigma}_{2}^{a}+\bar{\sigma}_{2}^{a} \wedge \bar{\sigma}_{2}^{b}\right)=0 .
\end{aligned}
$$


Similarly,

$$
\left\{\lambda_{1}^{k} \lambda_{2}^{2-k}\left(V_{2}^{a} \bar{\Omega}_{1}^{b}+V_{2}^{b} \bar{\Omega}_{1}^{a}\right), \lambda_{1}^{k^{\prime}} \lambda_{2}^{2-k^{\prime}} V_{i^{\prime}}^{m+1} \bar{\Omega}_{1}^{m+1}\right\}=\frac{-2 \bar{\mu} \mu^{k+k^{\prime}} \partial_{i^{\prime}}^{m+1}\left(\bar{\sigma}_{1}^{b} \wedge \bar{\sigma}_{1}^{a}+\bar{\sigma}_{1}^{a} \wedge \bar{\sigma}_{1}^{b}\right)}{\left(1+|\mu|^{2}\right)^{2}} .
$$

It is equal to zero. Next,

$$
\begin{aligned}
& \left\{\lambda_{1}^{k} \lambda_{2}^{2-k}\left(V_{1}^{a} \bar{\Omega}_{1}^{b}-V_{2}^{b} \bar{\Omega}_{2}^{a}\right), \lambda_{1}^{k^{\prime}} \lambda_{2}^{2-k^{\prime}} V_{i^{\prime}}^{m+1} \bar{\Omega}_{1}^{m+1}\right\} \\
= & \frac{\mu^{k+k^{\prime}}}{\left(1+|\mu|^{2}\right)^{2}} \partial_{i^{\prime}}^{m+1}\left(\bar{\sigma}_{1}^{b} \wedge L_{\partial_{1}^{a}} \bar{\sigma}_{1}^{m+1}-\bar{\sigma}_{2}^{a} \wedge L_{\partial_{2}^{b}} \bar{\sigma}_{1}^{m+1}\right) \\
= & 2 \bar{\mu} \frac{\mu^{k+k^{\prime}}}{\left(1+|\mu|^{2}\right)^{2}} \partial_{i^{\prime}}^{m+1}\left(\bar{\sigma}_{1}^{b} \wedge \bar{\sigma}_{2}^{a}+\bar{\sigma}_{2}^{a} \wedge \bar{\sigma}_{1}^{b}\right)=0 .
\end{aligned}
$$

Similarly,

$$
\left\{\lambda_{1}^{k} \lambda_{2}^{2-k}\left(V_{1}^{a} \bar{\Omega}_{1}^{b}-V_{2}^{b} \bar{\Omega}_{2}^{a}\right), \lambda_{1}^{k^{\prime}} \lambda_{2}^{2-k^{\prime}} V_{i^{\prime}}^{m+1} \bar{\Omega}_{2}^{m+1}\right\}=\frac{2 \mu^{k+k^{\prime}} \partial_{i^{\prime}}^{m+1}\left(\bar{\sigma}_{1}^{b} \wedge \bar{\sigma}_{2}^{a}+\bar{\sigma}_{2}^{a} \wedge \bar{\sigma}_{1}^{b}\right)}{\left(1+|\mu|^{2}\right)^{2}} .
$$

It is equal to zero. Finally,

$$
\begin{aligned}
& \left\{\lambda_{1}^{k} \lambda_{2}^{2-k}\left(V_{1}^{a} \bar{\Omega}_{2}^{b}+V_{1}^{b} \bar{\Omega}_{2}^{a}\right), \lambda_{1}^{k^{\prime}} \lambda_{2}^{2-k^{\prime}} V_{i^{\prime}}^{m+1} \bar{\Omega}_{2}^{m+1}\right\} \\
= & \frac{\mu^{k+k^{\prime}}}{\left(1+|\mu|^{2}\right)^{2}} \partial_{i^{\prime}}^{m+1}\left(\bar{\sigma}_{2}^{b} \wedge L_{\partial_{1}^{a}} \bar{\sigma}_{2}^{m+1}+\bar{\sigma}_{2}^{a} \wedge L_{\partial_{1}^{b}} \bar{\sigma}_{2}^{m+1}\right) \\
= & 2 \frac{\mu^{k+k^{\prime}}}{\left(1+|\mu|^{2}\right)^{2}} \partial_{i^{\prime}}^{m+1}\left(\bar{\sigma}_{2}^{b} \wedge \bar{\sigma}_{2}^{a}+\bar{\sigma}_{2}^{a} \wedge \bar{\sigma}_{2}^{b}\right)=0 .
\end{aligned}
$$

Similarly, the following term is equal to zero because

$$
\left\{\lambda_{1}^{k} \lambda_{2}^{2-k}\left(V_{2}^{a} \bar{\Omega}_{1}^{b}+V_{2}^{b} \bar{\Omega}_{1}^{a}\right), \lambda_{1}^{k^{\prime}} \lambda_{2}^{2-k^{\prime}} V_{i^{\prime}}^{m+1} \bar{\Omega}_{2}^{m+1}\right\}=\frac{-2 \mu^{k+k^{\prime}} \partial_{i^{\prime}}^{m+1}\left(\bar{\sigma}_{1}^{b} \wedge \bar{\sigma}_{1}^{a}+\bar{\sigma}_{1}^{a} \wedge \bar{\sigma}_{1}^{b}\right)}{\left(1+|\mu|^{2}\right)^{2}} .
$$

q. e. d.

All lemmas in this section together prove that when $\phi_{1}$ and $\phi_{2}$ are in $\mathcal{E}$, then there exist functions $g_{\tau} \in \Gamma^{0}$ such that $\left\{\phi_{1}, \phi_{2}\right\}=\sum \bar{\partial}\left(g_{\tau} \Upsilon_{\tau}\right)$. Suppose $\phi_{1}, \phi_{2}$ are in $\mathcal{E}$ and $h_{1}, h_{2}$ are in $\Gamma^{0}$. Since $\Upsilon_{\tau}(g)=0$ if $g \in \Gamma^{0}$,

$$
\left\{h_{1} \phi_{1}, h_{2} \phi_{2}\right\}=h_{1} h_{2}\left\{\phi_{1}, \phi_{2}\right\}=\sum_{\tau} h_{1} h_{2} \bar{\partial}\left(g_{\tau} \Upsilon_{\tau}\right)=\sum_{\tau}\left(h_{1} h_{2} \bar{\partial} g_{\tau}\right) \wedge \Upsilon_{\tau}
$$

Since $h_{1} h_{2} \bar{\partial} g_{\tau}$ is a $\bar{\partial}$-closed $(0,1)$-form on the Riemann sphere, it is $\bar{\partial}$-exact. Our proof of Proposition 1 is now completed. 


\subsection{Convergence}

Choose a Hermitian inner product on $\mathcal{E}$ such that the elements $\Upsilon_{\tau}$ form a Hermitian basis. On the Sobolev spaces $L_{k}^{2}\left(S^{2}\right)$ and $L_{k}^{2}\left(\Gamma^{(0,1)}\left(S^{2}\right)\right)$, we have the usual quadratic norm $\|\cdot\|_{k}$. Define a norm on $\|\cdot\|_{k}$ on $\Gamma^{0} \otimes \mathcal{E}$ by $\left\|\sum_{\tau} f_{\tau} \Upsilon_{\tau}\right\|_{k}^{2}=\sum_{\tau}\left\|f_{\tau}\right\|_{k}^{2}$. Similarly, define a norm $\|\cdot\|_{k}$ on $\Gamma^{(0,1)}\left(S^{2}\right) \otimes \mathcal{E}$ by $\left\|\sum_{\tau} \gamma_{\tau} \wedge \Upsilon_{\tau}\right\|_{k}^{2}=\sum_{\tau}\left\|\gamma_{\tau}\right\|_{k}^{2}$.

Lemma 10 There exists a constant $C_{1}$ such that if $f$ is a smooth function on $S^{2}$ with $\int_{S^{2}} f=0$, then $\|f\|_{k} \leq C_{1}\|\bar{\partial} f\|_{k-1}$.

Proof: The condition on $f$ implies that it is orthogonal to ker $\bar{\partial}^{*} \bar{\partial}$. By Schauder estimate [2, Appendix $\mathrm{H}$, Theorem 27], there exists a constant $c_{1}$ such that for all such $f,\|f\|_{k} \leq c_{1}\left\|\bar{\partial}^{*} \bar{\partial} f\right\|_{k-2}$.

Consider the first order elliptic operator $\bar{\partial}+\bar{\partial}^{*}: \Gamma^{(0,1)} \rightarrow \Gamma^{(0,0)} \oplus \Gamma^{(0,2)}$. Now $\bar{\partial}^{*} \bar{\partial} f=\left(\bar{\partial}+\bar{\partial}^{*}\right) \bar{\partial} f$. Since $\bar{\partial} f$ is orthogonal to $\operatorname{ker}\left(\bar{\partial}+\bar{\partial}^{*}\right)$, again by Schauder estimate there exists a constant $c_{2}$ such that for all such $f,\left\|\bar{\partial}^{*} \bar{\partial} f\right\|_{k-2} \leq c_{2}\|\bar{\partial} f\|_{k-1}$. The Lemma follows. q. e. d.

For the following lemma, see also [10, 5.118, 5.119].

Lemma 11 There exist constants $C_{2}$ and $C_{3}$ such that for all $\psi$ and $\phi$ in $\Gamma^{0} \otimes \mathcal{E}$,

$$
\begin{aligned}
\|\phi\|_{k} & \leq C_{2}\|\bar{\partial} \phi\|_{k-1} ; \\
\|\{\psi, \phi\}\|_{k} & \leq C_{3}\|\psi\|_{k+1}\|\phi\|_{k+1} .
\end{aligned}
$$

Proof: Since $\left\{\Upsilon_{\tau}: 1 \leq \tau \leq D\right\}$ is a Hermitian basis and elements are holomorphic, (50) follows from Lemma 10.

To prove (511), we assume that $\psi=g_{1} A_{1}, \phi=g_{2} A_{2}$ where $A_{1}$ and $A_{2}$ are one of the elements in the Hermitian basis $\left\{\Upsilon_{\tau}: 1 \leq \tau \leq D\right\}$. By Lemma 1, there exists $f_{\tau}$ such that $\left\{g_{1} A_{1}, g_{2} A_{2}\right\}=g_{1} g_{2} \sum_{\tau} \bar{\partial} f_{\tau} \wedge \Upsilon_{\tau}$. By definitions, $\left\|\left\{g_{1} A_{1}, g_{2} A_{2}\right\}\right\|_{k}^{2} \leq$ $\sum_{\tau}\left\|g_{1} g_{2} \bar{\partial} f_{\tau}\right\|_{k}^{2}$. Define

$$
c_{2}\left(A_{1}, A_{2}\right)=\sqrt{\max _{\ell \leq k, \tau} \sup \left|\nabla^{\ell} \bar{\partial} f_{\tau}\right|^{2}} .
$$

This constant depends on $A_{1}$ and $A_{2}$ because $f_{\tau}$ does. Define

$$
c_{2}=\max \left\{c_{2}\left(A_{1}, A_{2}\right): A_{1}, A_{2} \in\left\{\Upsilon_{\tau}: 1 \leq \tau \leq D\right\}\right\} .
$$

Then $\left\|g_{1} g_{2} \bar{\partial} f_{\tau}\right\|_{k} \leq c_{2}\left\|g_{1} g_{2}\right\|_{k}$. It is known [11, page 73] that there exists a constant $c_{3}$ such that $\left\|g_{1} g_{2}\right\|_{k} \leq c_{3}\left\|g_{1}\right\|\left\|_{k}\right\| g_{2} \|_{k}$ if $k \geq \operatorname{dim} S^{2}+1=3$. Combining all these inequalities, we have

$$
\begin{aligned}
\left\|\left\{g_{1} A_{1}, g_{2} A_{2}\right\}\right\|_{k} & \leq D c_{2}\left\|g_{1} g_{2}\right\|_{k} \leq D c_{2} c_{3}\left\|g_{1}\right\|_{k}\left\|g_{2}\right\|_{k} \\
& =D c_{2} c_{3}\left\|g_{1} A_{1}\right\|_{k}\left\|g_{2} A_{2}\right\|_{k} \leq D c_{2} c_{3}\left\|g_{1} A_{1}\right\|_{k+1}\left\|g_{2} A_{2}\right\|_{k+1} .
\end{aligned}
$$


In general, $\psi=\sum_{\tau} \psi_{\tau} \Upsilon_{\tau}, \phi=\sum_{\tau} \phi_{\tau} \Upsilon_{\tau}$. For $k \geq \operatorname{dim} S^{2}+1=3$, we obtain:

$$
\begin{aligned}
& \|\{\psi, \phi\}\|_{k}^{2}=\left\|\left\{\sum \psi_{\tau} \Upsilon_{\tau}, \sum \phi_{\rho} \Upsilon_{\rho}\right\}\right\|_{k}^{2} \leq \sum_{\tau, \rho}\left\|\left\{\psi_{\tau} \Upsilon_{\tau}, \phi_{\rho} \Upsilon_{\rho}\right\}\right\|_{k}^{2} \\
\leq & D^{2} c_{2}^{2} c_{3}^{2} \sum_{\tau, \rho}\left\|\psi_{\tau}\right\|_{k+1}^{2}\left\|\phi_{\rho}\right\|_{k+1}^{2}=D^{2} c_{2}^{2} c_{3}^{2}\left(\sum_{\tau}\left\|\psi_{\tau}\right\|_{k+1}^{2}\right)\left(\sum_{\rho}\left\|\phi_{\rho}\right\|_{k+1}^{2}\right) \\
= & D^{2} c_{2}^{2} c_{3}^{2}\left\|\sum \psi_{\tau} \Upsilon_{\tau}\right\|_{k+1}^{2} \cdot\left\|\sum \phi_{\rho} \Upsilon_{\rho}\right\|_{k+1}^{2}=D^{2} c_{2}^{2} c_{3}^{2}\|\psi\|_{k+1}^{2}\|\phi\|_{k+1}^{2}
\end{aligned}
$$

The proof is completed. q. e. d.

Lemma 12 Let $\Phi=\sum_{n} \Phi_{n} t^{n}$. Suppose that the coefficients $\Phi_{k}$ are vector-valued $(0,1)$-forms with the property that

$$
\bar{\partial} \Phi_{n+1}=-1 / 2 \sum_{i=1}^{n}\left\{\Phi_{i}, \Phi_{n+1-i}\right\}
$$

and suppose they satisfy the inequalities (50) and (51). Then for small enough $t, \Phi$ is convergent.

Proof: Take a power series $A(t)=\sum_{n=1}^{\infty} a_{n} t^{n}=\sum k \frac{(c t)^{n}}{n^{2}}$. It has nonzero radius of convergence. As seen in the proof of [10, Equation 5.116],

$$
\frac{1}{16 k} \sum_{i=1}^{n} a_{i} a_{n+1-i} \leq a_{n+1}
$$

Suppose that $\left\|\Phi_{n}\right\|_{k}^{2} \leq a_{n}$ up to some $n$. By the last lemma,

$$
\left\|\Phi_{n+1}\right\|_{k}^{2} \leq C_{2}^{2}\left\|\bar{\partial} \Phi_{n+1}\right\|_{k-1}^{2} \leq \frac{C_{2}^{2}}{4} \sum_{i=1}^{n}\left\|\left\{\Phi_{i}, \Phi_{n+1-i}\right\}\right\|_{k-1}^{2} \leq \frac{C_{3}^{2} C_{2}^{2}}{4} \sum_{i=1}^{n}\left\|\Phi_{n+1-i}\right\|_{k}^{2}\left\|\Phi_{i}\right\|_{k}^{2} .
$$

If we choose $\frac{1}{16 k}=\frac{C_{3}^{2} C_{2}^{2}}{4}$ and $c$ so that $\left\|\Phi_{1}\right\|^{2} \leq a_{1}=k c$, we obtain:

$$
\left\|\Phi_{n+1}\right\|_{k}^{2} \leq \frac{1}{16 k} \sum_{i=1}^{n}\left\|\Phi_{n+1-i}\right\|_{k}^{2}\left\|\Phi_{i}\right\|_{k}^{2} \leq \frac{1}{16 k} \sum_{i=1}^{n} a_{n+1-i} a_{i} \leq a_{n+1}
$$

So the Lemma follows by induction. The convergency of $A(t)$ is proved. q. e. d.

For any element $\Phi_{1}$ in $H^{1}\left(W, \mathcal{D}_{W}\right) \subset H^{1}\left(W, \Theta_{W}\right)$, Lemma 1 inductively determines solutions for the recursive formula $\bar{\partial} \Phi(t)+\frac{1}{2}\{\Phi(t), \Phi(t)\}=0$. Lemma 12 shows that there exists $\epsilon>0$ such that the power series $\Phi(t)=\Phi_{1} t+\Phi_{2} t^{2}+\ldots$ converges when $|t|<\epsilon$. It follows from Kodaira-Spencer theory that $\Phi_{1}$ is an infinitesimal deformation. Proof of Theorem 2 is completed. 


\subsection{Hypercomplex Deformations}

The integrability of $\Phi_{1}$ amounts to a deformation of quaternionic structures [15]. We complete our discussion on deformation of hypercomplex structures by showing that every quaternionic deformation of the given hypercomplex structure on $X$ is a hypercomplex deformation. This is Theorem 3 ,

The underlying smooth structure of the twistor spaces of the deformed quaternionic structures on $X$ remains to be $X \times S^{2}$. The underlying smooth structure of the twistor spaces of the deformed quaternionic structures on $T^{4 m}$ is $T^{4 m} \times S^{2}$. The natural projections $p_{W}$ and $p_{Z}$ from these spaces onto $S^{2}$ satisfy the identity $p_{W}=p_{Z} \circ \Psi$. When the twistor spaces are given the un-perturbed hypercomplex structures, the maps $p_{W}$ and $p_{Z}$ are holomorphic. We complete the proof of Theorem 3 by showing that for all local deformations found in the previous paragraph, the maps $p_{W}$ and $p_{Z}$ are holomorphic. They are denoted by $p$ in the previous sections. As a result of general twistor theory [15] [16], the deformed quaternionic manifolds are in fact hypercomplex manifolds.

To verify our claim on the open set $U_{2}$, we consider any distribution determined by the convergent power series $\Phi(t)=\sum_{n} \Phi_{n} t^{n}$ where $\Phi_{1}$ is in $H^{1}\left(W, \mathcal{D}_{W}\right) \subset \mathcal{E}$, and $\Phi_{n}$ is in $\Gamma^{0} \otimes \mathcal{E}$ for all $n \geq 2$. On the open set $U_{2}$ of the twistor space $W$, the space of $(0,1)$-vectors is spanned by $\left\{\bar{V}_{i}^{\alpha}, \frac{\partial}{\partial \bar{\mu}}\right\}$. Then $(0,1)$-vectors for the deformation family are spanned by

$$
\bar{V}_{k}^{\gamma}(t):=\bar{V}_{k}^{\gamma}+\Phi(t)\left(\bar{V}_{k}^{\gamma}\right)=\bar{V}_{k}^{\gamma}+\sum_{n} t^{n} \Phi_{n}\left(\bar{V}_{k}^{\gamma}\right)
$$

and $\frac{\partial}{\partial \bar{\mu}}$. To prove that $p_{W}$ is holomorphic for this family of complex structures, we verify that its differential sends $(0,1)$-vectors to $(0,1)$-vectors. Since $d p_{W}\left(\frac{\partial}{\partial \bar{\mu}}\right)=\frac{\partial}{\partial \bar{\mu}}$, our only concerns are on $d p_{W} \bar{V}_{k}^{\gamma}(t)$ which is equal to

$$
d p_{W}\left(\bar{V}_{k}^{\gamma}\right)+\sum t^{n} d p_{W}\left(\Phi_{n}\left(\bar{V}_{k}^{\gamma}\right)\right) .
$$

By construction, $\Phi_{n}$ is in the space $\mathcal{E}$ (44)). Therefore, $\Phi_{n}\left(\bar{V}_{k}^{\gamma}\right)$ is a linear combinations of $V_{\ell}^{\rho}$ for some $\ell$ and $\rho$. Therefore, it suffices to prove that $d p_{W}\left(\bar{V}_{k}^{\gamma}\right)=0$ for all $k$ and $\gamma$. Indeed, when $\gamma=m+1, \bar{V}_{k}^{\gamma}$ is given by coordinate vector fields $\frac{\partial}{\partial \bar{w}_{i}^{m+1}}$. As these vector fields are vertical with respect to the project $p_{W}$, they are in the kernel of the differential of $p_{W}$. Strictly speaking, when $1 \leq \gamma \leq m$, the element $\bar{V}_{k}^{\gamma}$ is contained in $H^{0}\left(W, \Psi^{*} \mathcal{D}\right)$. They could be interpreted as tangent vectors on $W$ only after a lifting process as described in our coboundary map computation in the proof of Theorem 2. In particular, as seen in (6) the lifting of $\frac{\partial}{\partial x_{j}}$ is $X_{j}$ and $\frac{\partial}{\partial y_{j}}$ is $Y_{j}$. Taking this lifting into account, we have the identity

$$
d p_{W}\left(\bar{V}_{k}^{\gamma}\right)=d p_{Z} \circ d \Psi\left(\bar{V}_{k}^{\gamma}\right)=d p_{Z}\left(\bar{V}_{k}^{\gamma}\right)
$$


where $\bar{V}_{k}^{\gamma}$ on the right hand side of the equation is now interpreted as a vector field on $Z$. On $Z, \bar{V}_{k}^{\gamma}$ is spanned by $\frac{\partial}{\partial \bar{w}_{j}^{\gamma}}$. As it is obviously in the kernel of $d p_{Z}$, we complete the proof that the map $p_{W}$ is holomorphic.

\subsection{Quaternionic deformations}

Computation in the last section suggests that we have to take a closer look at the parameter count for hypercomplex and quaternionic deformations and their relations. The relation could be manifested by the details in the following result.

Lemma 13 The coboundary maps $\delta_{0}$ and $\delta_{1}$ from the zero-th and first cohomology respectively in the induced cohomology sequence of

$$
0 \rightarrow \mathcal{D}_{W} \rightarrow \Theta_{W} \rightarrow p^{*} \mathcal{O}(2) \rightarrow 0
$$

are injective.

Proof: Due to Lemma 4, the following twisted k-forms form a basis for $H^{k}\left(W, p^{*} \mathcal{O}(2)\right)$.

$$
\lambda_{1}^{2+k-l} \lambda_{2}^{l} \bar{\Omega}_{1}^{\alpha_{1}} \wedge \cdots \wedge \bar{\Omega}_{1}^{\alpha_{i}} \wedge \bar{\Omega}_{2}^{\beta_{1}} \wedge \cdots \wedge \bar{\Omega}_{2}^{\beta_{j}}
$$

where $0 \leq l \leq 2+k$ and $1 \leq \alpha_{i}, \beta_{j} \leq m+1$.

Using the standard identification between quadratic polynomials and global holomorphic vector fields on $\mathbf{C P}^{1}$, i.e. $\left(a \lambda_{1}^{2}+b \lambda_{1} \lambda_{2}+c \lambda_{2}^{2}\right) \mapsto\left(a \mu^{2}+b \mu+c\right) \frac{\partial}{\partial \mu}$, we consider the above sections as holomorphic 1-forms on the twistor space with values in the tangent bundle of $\mathbf{C P}^{1}$. Then the above sections are identified to

$$
s=\mu^{2+k-l} \frac{\partial}{\partial \mu} \bar{\sigma}_{1}^{\alpha_{1}} \wedge \cdots \wedge \bar{\sigma}_{1}^{\alpha_{i}} \wedge \bar{\sigma}_{2}^{\beta_{1}} \wedge \cdots \wedge \bar{\sigma}_{2}^{\beta_{j}} .
$$

To find the image of $s$ through the coboundary map $\delta_{k}$, we lift the 1 -forms by pullbacks and the vector field is lifted to $W$. It defines a smooth lifting $\hat{s}$. We use the Chern connection $\nabla$ on the holomorphic tangent bundle on $W$ to define the $\bar{\partial}$ operator $\bar{\partial}^{\nabla}$. Then $\delta_{k} s$ is represented uniquely by the cohomology class of $\bar{\partial} \hat{s}$. Since the function $\mu^{\ell+2-l}$ and the forms $\bar{\sigma}_{1}^{\alpha}$ and $\bar{\sigma}_{2}^{\beta}$ are holomorphic,

$$
\bar{\partial}^{\nabla} \hat{s}=\mu^{2+k-l}\left(\bar{\partial}^{\nabla} \frac{\partial}{\partial \mu}\right) \wedge \bar{\sigma}_{1}^{\alpha_{1}} \wedge \cdots \wedge \bar{\sigma}_{1}^{\alpha_{i}} \wedge \bar{\sigma}_{2}^{\beta_{1}} \wedge \cdots \wedge \bar{\sigma}_{2}^{\beta_{j}} .
$$

Due to an observation of Gauduchon [4], for any (0,1)-vector $X$, and (1,0)-vector $Y$, $\bar{\partial}_{X}^{\nabla} Y=[X, Y]^{1,0}$. Using (133) and (134), we find that

$$
\left[\bar{\partial}_{1}^{\alpha}, \frac{\partial}{\partial \mu}\right]^{1,0}=-\frac{\partial_{2}^{\alpha}}{1+|\mu|^{2}}, \quad\left[\bar{\partial}_{2}^{\alpha}, \frac{\partial}{\partial \mu}\right]^{1,0}=\frac{\partial_{1}^{\alpha}}{1+|\mu|^{2}},
$$


It follows that $\bar{\partial}^{\nabla}\left(\frac{\partial}{\partial \mu}\right)$ is equal to

$$
\begin{aligned}
& -\frac{d \bar{w}_{1}^{\alpha} \otimes \partial_{2}^{\alpha}}{1+|\mu|^{2}}+\frac{d \bar{w}_{2}^{\alpha} \otimes \partial_{1}^{\alpha}}{1+|\mu|^{2}}+\frac{w_{1}^{\alpha}-\mu \bar{w}_{2}^{\alpha}}{\left(1+|\mu|^{2}\right)^{2}} d \bar{\mu}_{1} \otimes \partial_{1}^{\alpha}+\frac{w_{2}^{\alpha}+\mu \bar{w}_{1}^{\alpha}}{\left(1+|\mu|^{2}\right)^{2}} d \bar{\mu}_{1} \otimes \partial_{2}^{\alpha} \\
= & -\frac{d \bar{w}_{1}^{\alpha} \otimes \partial_{2}^{\alpha}}{1+|\mu|^{2}}+\frac{d \bar{w}_{2}^{\alpha} \otimes \partial_{1}^{\alpha}}{1+|\mu|^{2}}-\frac{\bar{z}_{2}^{\alpha}}{\left(1+|\mu|^{2}\right)^{2}} d \bar{\mu}_{1} \otimes \partial_{1}^{\alpha}+\frac{\bar{z}_{1}^{\alpha}}{\left(1+|\mu|^{2}\right)^{2}} d \bar{\mu}_{1} \otimes \partial_{2}^{\alpha} \\
= & \sum_{\alpha}\left(\bar{\sigma}_{2}^{\alpha} \otimes \partial_{1}^{\alpha}-\bar{\sigma}_{1}^{\alpha} \otimes \partial_{2}^{\alpha}\right) .
\end{aligned}
$$

Therefore, $\bar{\partial}_{k}^{\nabla} \hat{s}$ is equal to

$$
\begin{aligned}
& \mu^{2+k-l} \sum_{\alpha}\left(\bar{\sigma}_{2}^{\alpha} \otimes \partial_{1}^{\alpha}-\bar{\sigma}_{1}^{\alpha} \otimes \partial_{2}^{\alpha}\right) \wedge \bar{\sigma}_{1}^{a_{1}} \wedge \cdots \wedge \bar{\sigma}_{1}^{a_{i}} \wedge \bar{\sigma}_{2}^{b_{1}} \wedge \cdots \wedge \bar{\sigma}_{2}^{b_{j}} \\
= & \lambda_{1}^{2+k-l} \lambda_{2}^{l} \sum_{\alpha}\left(\bar{\Omega}_{2}^{\alpha} \otimes \frac{1}{\lambda_{2}} \partial_{1}^{\alpha}-\bar{\Omega}_{1}^{\alpha} \otimes \frac{1}{\lambda_{2}} \partial_{2}^{\alpha}\right) \wedge \bar{\Omega}_{1}^{a_{1}} \wedge \cdots \wedge \bar{\Omega}_{1}^{a_{i}} \wedge \bar{\Omega}_{2}^{b_{1}} \wedge \cdots \wedge \bar{\Omega}_{2}^{b_{j}} \\
= & \lambda_{1}^{2+k-l} \lambda_{2}^{l} \sum_{\alpha}\left(\bar{\Omega}_{2}^{\alpha} \otimes V_{1}^{\alpha}-\bar{\Omega}_{1}^{\alpha} \otimes V_{2}^{\alpha}\right) \wedge \bar{\Omega}_{1}^{a_{1}} \wedge \cdots \wedge \bar{\Omega}_{1}^{a_{i}} \wedge \bar{\Omega}_{2}^{b_{1}} \wedge \cdots \wedge \bar{\Omega}_{2}^{b_{j}} .
\end{aligned}
$$

This map is injective when $k=0,1$. In particular,

$$
\delta_{0}\left(\lambda_{1}^{2-l} \lambda_{2}^{l}\right)=\lambda_{1}^{2-l} \lambda_{2}^{l} \sum_{\alpha}\left(\bar{\Omega}_{2}^{\alpha} \otimes V_{1}^{\alpha}-\bar{\Omega}_{1}^{\alpha} \otimes V_{2}^{\alpha}\right)
$$

The proof is now completed. q. e. d.

As a consequence of the last lemma, the following sequence is exact

$$
0 \rightarrow H^{0}\left(W, p^{*} \mathcal{O}(2)\right) \rightarrow H^{1}\left(W, \mathcal{D}_{W}\right) \rightarrow H^{1}\left(W, \Theta_{W}\right) \rightarrow 0 .
$$

It confirms, but does not prove our result in the last section, that every quaternionic deformation is a hypercomplex deformation. The new information in the above exact sequence is, that for every hypercomplex deformation parameter, there is a threedimensional hypercomplex deformation within one quaternionic class. This parameter space is contributed by $H^{0}\left(W, p^{*} \mathcal{O}(2)\right) \cong S^{2} \mathbf{C}^{2}$. Since $H^{1}\left(W, \Theta_{W}\right)$ is the virtual parameter space for complex structures on the twistor space $W$, its real subspace with respect to the real structure $\tau$ is the parameter space for quaternionic structure on $X$, it completes the proof of Theorem 4 .

\subsection{Deformations of Torus}

During the course of our computation, significant amount of information on deformation of the hypercomplex structure on the torus $T^{4 m}$ as a quotient of the quaternion module $\mathbf{H}^{m}$ is revealed. By Lemma 3, the virtual parameter space for hypercomplex deformation on the torus $T^{4 m}$ is the real part of the complex vector 
space $\mathfrak{t}^{1,0} \otimes \mathfrak{t}^{*(0,1)} \otimes S^{2} \mathbf{C}^{2}$. Its real dimension is equal to complex dimension; i.e. $(2 m)^{2} \times 3=12 m^{2}$. To prove integrability, we apply the method in Section 4.1 with the space $\mathcal{E}$ replaced by $H^{1}\left(Z, \mathcal{D}_{Z}\right)$. In this case, we do not need the work in Section 4.3 to conclude that every deformation generated by $H^{1}\left(Z, \mathcal{D}_{Z}\right)$ is hypercomplex as the power series generated in Section 4.1 has values in $H^{1}\left(Z, \mathcal{D}_{Z}\right)$. Finally, the exact sequence (60) has its counter part on $Z$. Therefore, the parameter space for quaternionic deformation on $T^{4 m}$ is equal to $12 m^{2}-3$.

In Section 2.2, we construct a hypercomplex structure on $\mathbf{R}^{4 m}$ by left multiplication of unit quaternions $i, j$ and $k$ on $\mathbf{H}^{m}$. As seen in Section 2.1, the torus $T^{4 m}$ is the quotient of $\mathbf{R}^{4 m}$ with respect to the lattice group $\phi(\Gamma) \cong\left(\mathbf{Z}^{4 m},+\right)$. The right multiplication by a generic element in $\operatorname{GL}(4 m, \mathbf{R})$ changes the hypercomplex structure on $T^{4 m}$ by choosing different identification from $\mathbf{R}^{4 m}$ to $\mathbf{H}^{m}$. The isotropy subgroup with respect to the quaternion basis $\left\{Q_{a}, 1 \leq a \leq m\right\}$ is $\mathrm{GL}(m, \mathbf{H})$. Therefore, the (coarse topological) moduli space of hypercomplex structures is $\operatorname{Aut}\left(\mathbf{Z}^{4 m}\right) \backslash \mathrm{GL}(4 m, \mathbf{R}) / \mathrm{GL}(m, \mathbf{H})$ where $\operatorname{Aut}\left(\mathbf{Z}^{4 m}\right)$ is the discrete subgroup of $G L(4 m, \mathbf{R})$ consisting of automorphisms of the lattice. Furthermore, left multiplication of $\operatorname{Sp}(1)$ changes the hypercomplex structure but keeps the quaternionic structure. Therefore, the (coarse topological) moduli space of quaternionic structures is $\operatorname{Aut}\left(\mathbf{Z}^{4 m}\right) \backslash \mathrm{GL}(4 m, \mathbf{R}) / \mathrm{GL}(m, \mathbf{H}) \mathrm{Sp}(1)$. Our computation on the dimension of moduli proves that these spaces are the entire connected component of the moduli space of hypercomplex deformations and quaternionic deformations respectively.

\section{Moduli of Invariant Hypercomplex Structures}

By invariant hypercomplex structure we mean a triple of left-invariant complex structures on $X$ with the usual relations. Such triple is determined by its values at the identity. The first observation in this section is due to the power series calculations in Section 4.1.

Proposition 2 Any small deformation of the hypercomplex structure on $X$ consists of invariant structures.

Proof: From the calculations in the proof of Proposition 1, Section 4.1 we can conclude that in the series $\Phi(t)=\sum_{n} \Phi_{n} t^{n}$ all terms $\Phi_{n}$ belong to $\Gamma_{0} \otimes \mathcal{E}$. In particular for every fixed $t_{0}, \Phi\left(t_{0}\right)$ is in the same space. Then substituting $\left(\lambda_{1}, \lambda_{2}\right)$ with $(1,0),(0,1)$ and $(i, 1)$ we obtain elements in $\mathcal{E}$. They determine invariant complex structures by identifying $\mathcal{E}$ with a subspace of the space of sections in $T^{(1,0) X} \otimes T^{*(0,1)}$. Each section is identified with the image of the bijective linear map $I d+\Phi: T_{I}^{(0,1)} X \rightarrow T_{J}^{(0,1)} X$ and the deformed structure $J$ is defined from here. Since the elements of $\mathcal{E}$ are given 
by invariant sections, the deformed structures are invariant. Then the three invariant complex structures give rise to an invariant hypercomplex structure. q. e. d.

Further in this section we investigate the deformations of the hypercomplex structures on $\left(H_{4 m+1} \times \mathbf{R}^{3}\right) / \Gamma$ arising from the deformations of the lattice $\Gamma$. As we will see this space differs from the space of invariant hypercomplex structures.

From Theorem [ 6 the left invariant hypercomplex structure defined in Section 2.2 is equivalent to the canonical one on $\mathbf{H}^{m}$.

Now if $\Gamma^{\prime}$ is another lattice in $H_{4 m+1} \times \mathbf{R}^{3}$ such that the quotient spaces $H_{4 m+1} \times$ $\mathbf{R}^{3} / \Gamma$ and $H_{4 m+1} \times \mathbf{R}^{3} / \Gamma^{\prime}$ are isomorphic as hypercomplex manifolds, then $\Gamma$ and $\Gamma^{\prime}$ are isomorphic. According to ([17], Theorem 2.11, Corollary 2) the isomorphism between the two cocompact subgroups is uniquely extended to an automorphism $\bar{\Upsilon}$ of $H_{4 m+1} \times \mathbf{R}^{3}$. Then by the functorial property of the exponential map there is a unique Lie algebra automorphism $\Upsilon$ of $\mathfrak{h}_{4 m_{+} 1} \oplus \mathfrak{t}^{3}$ defined by $\bar{\Upsilon}$. In particular the deformations arising from deformation of the lattice lie in the orbit of the automorphism group of $\mathfrak{h}_{4 m_{+}+1} \oplus \mathfrak{t}^{3}$ containing the standard hypercomplex structure. Bellow we consider the form of $\Upsilon$.

Proposition 3 Let $\left\{Z, E_{1}, E_{2}, E_{3}, X_{2 a-1}, X_{2 a}, Y_{2 a-1}, Y_{2 a}\right\}$ be an ordered basis for $\mathfrak{h}_{4 m+1} \times$ $\mathbf{R}^{3}$. The automorphism group of $\mathfrak{h}_{4 m+1} \oplus \mathfrak{t}^{3}$ consists of elements $\Upsilon$ leaving the center invariant of the form:

$$
\Upsilon=\left(\begin{array}{cc}
A & B \\
0 & C
\end{array}\right)
$$

where $A$ is in Endc $\oplus \operatorname{Hom}\left(\mathfrak{t}^{3}, \mathfrak{c} \oplus \mathfrak{t}^{3}\right)$, and $B$ is in $H o m\left(\mathfrak{h}_{4 m+1} \oplus \mathfrak{t}^{3}, \mathfrak{c} \oplus \mathfrak{t}^{3}\right)$. Moreover, $C$ is a matrix preserving the symplectic form in $\mathbf{R}^{4 m}$ up to a constant.

Proof: The Lie brackets is

$$
\left[V, V^{\prime}\right]=-2 \omega\left(V, V^{\prime}\right) Z
$$

for the skew form $\omega$ with $K e r \omega=\operatorname{span}\left\{Z, E_{1}, E_{2}, E_{3}\right\}$ and $\omega\left(V, V^{\prime}\right)=2\left(-y_{i} x_{i}^{\prime}+x_{i} y_{i}^{\prime}\right)$ where $V=\left(z, e_{j}, x_{i}, y_{i}\right)$ and $V^{\prime}=\left(z^{\prime}, e_{j}^{\prime}, x_{i}^{\prime}, y_{i}^{\prime}\right)$ in the given basis. Since $\Upsilon$ preserves the center and the direct sum $\mathfrak{h}_{4 m+1} \oplus \mathfrak{t}^{3}, \Upsilon(Z)=S_{0} Z$ for some constant $S_{0}$. From the form of the brackets above we obtain $\omega\left(\Upsilon(V), \Upsilon\left(V^{\prime}\right)\right)=S_{0} \omega\left(V, V^{\prime}\right)$. q. e. d.

For further use denote the set of all $\Upsilon$ for which $\omega\left(\Upsilon(V), \Upsilon\left(V^{\prime}\right)\right)=S \omega\left(V, V^{\prime}\right)$ for a fixed $S$ as $C_{S} S p(2 m, \mathbf{R})$

Corollary 1 The dimension of the space of automorphisms in Proposition 2 is $13+$ $18 m+8 m^{2}$ 
Proof: We calculate the dimensions of the three blocks. The $4 \times 4$ block in the upperleft corner consisting of $S$ 's has dimension 13 . The upper-right block with $F_{a}$ 's and $G_{a}$ 's has dimension $4 \times 4 m=16 m$. Finally the set of symplectic transforms up to a constant $C S p(2 m, \mathbf{R})$ has the same dimension as the group $S p(2 m, \mathbf{R})$ which is $2 \mathrm{~m}(4 \mathrm{~m}+1)$. When we sum up the dimensions the result follows.

q. e. d.

For any automorphism $\Upsilon$ of $h_{4 m+1} \oplus \mathfrak{t}^{3}$, we define hypercomplex structure $\mathcal{I}_{\Upsilon}$ by $\Upsilon^{-1} \circ \mathcal{I} \circ \Upsilon$ with the new basis $\Upsilon$. Then it will induce hypercomplex structure defined on $\left(H_{4 m+1} \times \mathbf{R}^{3}\right) / \bar{\Upsilon}(\Gamma)$ via factorisation. Now we have to consider which $\Upsilon$ give rise to equivalent structures on the factor-space.

Proposition 4 The hypercomplex spaces $\left(H_{4 n+1} \times \mathbf{R}^{3}\right) / \Gamma$ and $\left(H_{4 n+1} \times \mathbf{R}^{3}\right) / \bar{\Upsilon}(\Gamma)$ are equivalent if and only if:

$$
\begin{aligned}
& A=s I, s \neq 0 \\
& B \in C S p(2 m, \mathbf{R}) \cap G L(m, \mathbf{H})=C S p(m) \\
& C=\left(C_{1} C_{2} \ldots C_{n}\right), C_{i}=\left(\begin{array}{cccc}
\alpha_{2 i-1} & \alpha_{2 i} & \beta_{2 i-1} & \beta_{2 i} \\
-b_{i} & a_{i} & -d_{i} & c_{i} \\
-c_{i} & d_{i} & a_{i} & -b_{i} \\
-d_{i} & -c_{i} & b_{i} & a_{i}
\end{array}\right)
\end{aligned}
$$

In particular the dimension of the group of hypercomplex automorphisms arising in this way is $1+9 m+2 m^{2}$.

Proof: Any hypecomplex automorphism of $H_{4 m+1} \times \mathbf{R}^{3} / \Gamma$ gives rise to automorphism on $H_{4 m+1} \times \mathbf{R}^{3}=\mathbf{H}^{n+1}$ which interchange the actions of $\Gamma$ and $\Gamma^{\prime}=\bar{\Upsilon}(\Gamma)$. But any hypercomplex automorphism of $\mathbf{H}^{n+1}$ is an affine transformation by a theorem of Ehresman. So $(A, \mathbf{v}): \mathbf{q} \rightarrow A \mathbf{q}+\mathbf{v}$ with $A \in G L(n+1, \mathbf{H})$ is a general form of a hypercomplex automorphism. Then to verify the proposition we have to check that if

$$
(A, \mathbf{v}) \circ \gamma=\gamma^{\prime} \circ(A, \mathbf{v})
$$

with $\gamma \in \Gamma$ and $\gamma^{\prime}=\bar{\Upsilon}(\gamma) \in \bar{\Upsilon}(\Gamma)$, then $\Upsilon$ has the proposed form.

The group multiplication is expressed in terms of $\omega$ as

$$
x * x^{\prime}=x+x^{\prime}+\omega\left(x, x^{\prime}\right) z
$$

where $z=(1,0,0, \ldots, 0)$ is the center generator. Then the condition above becomes $A(\gamma * \mathbf{q})+\mathbf{v}=\gamma^{\prime} *(A \mathbf{q}+\mathbf{v})$. It follows that

$$
A \gamma+\omega(\gamma, \mathbf{q}) A z=\gamma^{\prime}+\omega\left(\gamma^{\prime}, A \mathbf{q}+\mathbf{v}\right) z
$$


Substituting $\mathbf{q}=0$, we have

$$
A \gamma=\gamma^{\prime}+\omega\left(\gamma^{\prime}, \mathbf{v}\right) z
$$

Substitute the last formula back, we have

$$
\omega\left(\gamma^{\prime}, A \mathbf{q}\right) z=\omega(\gamma, \mathbf{q}) A z \text { and } A \gamma=\gamma^{\prime}+\omega\left(\gamma, A^{-1} \mathbf{v}\right) A z .
$$

It follows that

$$
\bar{\Upsilon}(\gamma)=\gamma^{\prime}=A \gamma-\omega\left(\gamma, A^{-1} \mathbf{v}\right) A z .
$$

As both $A$ and $\omega$ are linear, $\bar{\Upsilon}$ is linear. The above formula is applicable to any element in $H_{4 m+1} \times \mathbf{R}^{3}$. It also shows that $(A, \mathbf{v})$ uniquely determines $\bar{\Upsilon}$.

Now using the identification of $\Upsilon$ and $\bar{\Upsilon}$ via the exponential map we are going to obtain the form of $\Upsilon$ in the proposition. Note that A is simultaneuosly Lie-algebra automorphism described in Proposition 2 and an element of $G L(m+1, \mathbf{H})$.

We first consider the case $\mathbf{v}=0$. Here we have to characterize the matrices $A$ as above. The group $G L(m+1, \mathbf{H})$ is identified with the group preserving the hypercomplex structure. The structures $I_{1}, I_{2}, I_{3}$ are formed by $4 \times 4$-blocks along the diagonal of the following type:

$$
J_{1}=\left(\begin{array}{cccc}
0 & -1 & 0 & 0 \\
1 & 0 & 0 & 0 \\
0 & 0 & 0 & -1 \\
0 & 0 & 1 & 0
\end{array}\right)
$$

and

$$
J_{2}=\left(\begin{array}{cccc}
0 & 0 & -1 & 0 \\
0 & 0 & 0 & 1 \\
1 & 0 & 0 & 0 \\
0 & -1 & 0 & 0
\end{array}\right)
$$

and $J_{3}=J_{1} J_{2}$. Then when we divide the matrix of $\Upsilon$ in $4 \times 4$-blocks, then each block should represent a matrix which commute with $J_{1}$ and $J_{2}$ above. The general form of a $4 \times 4$ matrix which commutes with $J_{1}$ and $J_{2}$ is

$$
B=\left(\begin{array}{cccc}
a & b & c & d \\
-b & a & -d & c \\
-c & d & a & -b \\
-d & -c & b & a
\end{array}\right)
$$


for some numbers $a, b, c, d$. The diagonal blocks additionally have nonzero determinant.

Now the upper-left corner of $A$ has first column with zero's except $S_{0}$, so it is proportional to the identity. The lower-right corner is $4 m \times 4 m$ consisting of $P_{2 a-1}, P_{2 a}, Q_{2 a-1}, Q_{2 a}$ is an element of $C_{S_{0}} S p(m)$. Finally the upper-right $4 \times 4 \mathrm{~m}$ corner consists of $4 \times 4$ matrices $\left(F_{2 i-1} \ldots G_{2 i}\right)$ for $i=1, \ldots m$. Here $i$ represents the number of the block. Then all matrices have the form of $B$ above.

Now we have to consider the case $\mathbf{v} \neq 0$. In the formula $\gamma \rightarrow A \gamma-\omega\left(\gamma, A^{-1} \mathbf{v}\right) A z$ we have that the second term is always proportional to $z$ i.e. $\omega\left(\gamma, A^{-1} \mathbf{v}\right) A z=C z$. By varying $v$ we may choose any constant of proportion $C$. Now when $\gamma$ is chosen to be any of the basic vectors the formula above changes an element of the first row of $\Upsilon$ because of this term. Thus the first row of $\Upsilon$ is arbitrary. With this we have the form of the matrix $\left(F_{2 i-1}, F_{2 i}, G_{2 i-1}, G_{2 i}\right)$ in the proposition since the center is the first element in the basis. From here the proposition follows.

q. e. d.

Corollary 2 The dimensions of automorphisms in Proposition 3 is $1+9 m+2 m^{2}$

Proof: The calculation is similar to that in the previous Corollary. This time the dimension of upper-left corner is 1 , because the matrix is proportional to identity. As efore the dimension of the set $C S p(m)$ is the same as the dimension of $S p(m)=$ $S p(m, \mathbf{C}) \cap U(2 m)=m(2 m+1)$. Finally the block with $F_{a}$ 's and $G_{a}$ 's has dimension $2 \times 4 m=8 m$. This gives the corollary. q. e. d.

From the above we obtain that the space of effective parameters for the deformations of the hypercomplex structures on $X$ arising from deformation of the lattice is $\frac{G}{H}$ where $G$ is the group of all $\Upsilon$ 's in Proposition 2 while $H$ is the group described in the Proposition 3. The dimension of this space is $12+9 m+6 m^{2}$. Now $\operatorname{dim}_{C} H^{1}\left(W, D_{W}\right)=12+11 m+6 m^{2}$ and $H^{0}\left(W, D_{W}\right)$ is generated by the elements in the center of $X$. To count the independent parameters in the deformation space we need the following:

Lemma 14 All elements in the basis of $H^{1}\left(W, D_{W}\right)$ from (43) are invariant under hypercomplex transformations.

Proof: The elements in $H^{0}\left(W, D_{W}\right)$ are described in Lemma 5. In particular the whole space is spanned by linear combinations of the vectors in the center $\left(Z, E_{1}, E_{2}, E_{3}\right)$ with coefficients depending on the fiber coordinate $\mu$. More precisely $H^{0}\left(W, D_{W}\right)=$ $\operatorname{span}\left\{W_{k}^{m+1}=I_{k} Z-i I_{\vec{a}} I_{k} Z\right\}$ at the point $\vec{a}$. Then $W_{k}^{m+1}$ are expressed locally as in (36) in terms of the local vector fields $\partial_{i}^{m+1}$. The $\partial_{i}^{m+1}$ themselves are defined in (34). 
In particular there is no $\frac{d}{d \mu}$ involved. The space $H^{1}\left(W, D_{W}\right)$ is a subspace of the span of $\lambda_{1}^{k} \lambda_{2}^{2-k} V_{i}^{a} \bar{\Omega}_{j}^{b}$. Again as in the calculation for the Nijenhuis bracket the elements are expressed locally in terms of $\frac{\mu^{k}}{|\mu|^{2}+1} \partial_{i}^{\alpha} \bar{\sigma}_{j}^{\beta}$ for $k=0,1,2$. Here $\mu$ is inessential too.

Now we have to check that $\mathcal{L}_{X}(A)=0$ for $X \in H^{0}$ and $A \in H^{1}$. The formula we need is:

$$
\mathcal{L}_{X}(\alpha \otimes Y)=\left(\mathcal{L}_{X} \alpha\right) \otimes Y+\alpha \otimes\left(\mathcal{L}_{X} Y\right)
$$

Now we take $X$ to be any vector which is linear combination of $\left(Z, E_{1}, E_{2}, E_{3}\right)$ with coefficients depending on $\mu$. Then $\mathcal{L}_{X} V_{i}^{a}=0$ for any $V_{i}^{a}$ - here $\mu$ is a constant for the differentiations. Moreover using

$$
\mathcal{L}_{\partial_{i}^{m+1}}\left(\bar{\sigma}_{j}^{\alpha}\right)\left(\bar{\partial}_{k}^{\beta}\right)=\partial_{i}^{m+1}\left(\bar{\sigma}_{j}^{\alpha}\left(\bar{\partial}_{k}^{\beta}\right)\right)-\bar{\sigma}_{j}^{\alpha}\left(\left[\partial_{i}^{m+1}, \bar{\partial}_{k}^{\beta}\right]\right)=0
$$

we see that $\mathcal{L}_{X} \bar{\Omega}_{j}^{b}(Y)=0$ for $X$ in $H^{0}$ because $Y$ is a combination of vectors $\partial_{k}^{\beta}$.

q. e. d.

So by the Lemma all small deformations of the structures are again invariant structures by Cathelineau's theory and there are no equivalent deformations in $H^{1}$.

Corollary 3 There are deformations of the hypercomplex structure on $X$ which do not arise from a deformation of the lattice $\Gamma$.

This combined with Proposition 2 proves Theorem 5 from the Introduction.

Remark 1 We note a similar phenomena for the complex deformations of the 3dimensional complex Heisenberg group $G$. The local moduli space around its canonical complex structure is calculated by [13] and is 6 dimensional. The connected component of the invariant complex structures at this point is again 6-dimensional, but the orbit of the complex structure under the action of the automorphism group of $G$ is only 2 dimensional [19]. Since every two lattices are equivalent under an automorphism of $G$, it follows that there are deformations of the complex structure, which do not arise from deformations of the lattice, unlike the case of the complex torus. This also led Nakamura [13] to the conclusion that a small deformation of a complex parallelizable manifold is not necessary complex parallelizable. What we proved in this section is that similar fenomena holds for the small deformations of the hypercomplex structure of $X$.

Remark 2 One could notice that above we included identifications of the structures which arise from affine transformations. This identifies additionally some structures in the orbit of the automorphism group, since it acts only by linear transformations. So the number of effective parameters for the small deformations is less then the 
dimension of the orbit. Similar fenomena appears in the complex deformations of $H_{2 n+1} \times R / \Gamma$ as described in [6]. Due to the translation factor, there are no "offdiagonal" deformations. The difference in the hypercomplex case is that this amounts to all identifications because of the properties of hypercomplex automorphisms of $\mathbf{H}^{n}$. In the complex deformations case, this follows from the Kuranishi theory [12].

\section{References}

[1] M. L. Barberis. Abelian hypercomplex structures on central extensions of H-type Lie algebras, J. Pure Appl. Algebra, 158 (2001), 15-23.

[2] A. Besse. Einstein Manifolds, Ergebnisse der Mathematik und ihrer Grenzgebiete, 3. Folge 10, Springer-Verlag, New York 1987.

[3] M. L. Barberis \& I. Dotti Miatello Hypercomplex structures on a class of solvable Lie groups, Quart. J.Math. Oxford (2) 47 (1996), 389-404.

[4] P. Gauduchon. Hermitian connections and Dirac operators, Bollettino U.M.I., 11B (1997) 257-288.

[5] W. M. Goldman \& J. J. Wilson. The homotopy invariance of the Kuranishi space, Ill. J. Math. 34 (1990) 337-367.

[6] G. Grantcharov, C. McLaughlin, H. Pedersen \& Y. S. Poon. Deformations of Kodaira Manifolds, Glasg. Math. J. 46 (2004) 259-281..

[7] E. Horikawa. On deformations of holomorphic maps I, J. Math. Soc. Japan, 25 (1973) 372-396. II, J. Math. Soc. Japan, 26 (1974) 647-667.

[8] D. Joyce. Compact hypercomplex and quaternionic manifolds, J. Differential Geom. 35 (1992) 743-761.

[9] D. Joyce. Manifolds with many complex structures, Quart. J. Math. 46 (1995), 169-184.

[10] K. Kodaira Complex Manifolds and Deformation of Complex Structures, Springer-Verlag, New York, 1986.

[11] M. Kuranishi. Deformations of Compact Complex Manifolds, Séminaire de Mathématiques Supérieures, Les Presses de l'université de Montréal 1971.

[12] C.McLaughlin, H.Pedersen, Y.S.Poon, S.Salamon Deformation of 2-step Nilmanifolds with Abelian Complex Structures, preprint, math.DG/0402069. 
[13] I. Nakamura Complex parallelizable manifolds and their small deformations, J. Diff.Geom, 10 (1975) 85-112.

[14] A. Fröhlihcher \& A. Nijenhuis Theory of vector-valued forms I. Derivations in the graded ring of differential forms, Nedrl. Akad. Wetensch. Proc. Ser. A 59 (1956) 338-359.

[15] H. Pedersen \& Y. S. Poon. Twistorial construction of quaternionic manifolds, Proc. VI International Coll. Differential Geo., Santiago, Spain 1988. Univ. Santiago de Compostela, (1989).

[16] H. Pedersen \& Y. S. Poon. Deformations of hypercomplex structures, J. reine angew. Math. 499 (1998) 81-99.

[17] Raghunatan, Discrete subgroups of Lie groups, Springer (1971)

[18] S. M. Salamon. Differential geometry of quaternionic manifolds, Ann. scient. Éc. Norm. Sup. $4^{e}, 19$ (1986) 31-55.

[19] S. M. Salamon,Complex structures on nilpotent Lie algebras , J.Pure Appl.Algebra, 157(2001) 311-333 